\title{
Modeling and Simulation of Transverse Free Vibration Analysis of a Rectangular Plate with Cutouts Using Energy Principles
}

\author{
Shuangxia Shi, ${ }^{1,2}$ Bin Xiao $\mathbb{D}^{1},{ }^{1}$ Guoyong Jin $\mathbb{D},{ }^{2}$ and Chao Gao ${ }^{1}$ \\ ${ }^{1}$ School of Energy and Power Engineering, Northeast Electric Power University, Jilin 132012, China \\ ${ }^{2}$ College of Power and Energy Engineering, Harbin Engineering University, Harbin 150001, China \\ Correspondence should be addressed to Bin Xiao; dr.binxiao@yahoo.com
}

Received 14 December 2017; Revised 8 March 2018; Accepted 27 March 2018; Published 21 June 2018

Academic Editor: Md Abdul Halim Miah

Copyright (c) 2018 Shuangxia Shi et al. This is an open access article distributed under the Creative Commons Attribution License, which permits unrestricted use, distribution, and reproduction in any medium, provided the original work is properly cited.

\begin{abstract}
A modeling method is proposed for the vibration characteristics of rectangular plates with cutouts having variable size. Different from the existing modeling method by considering the cutout as an extremely thin part of the plate, the energy principles in conjunction with Rayleigh-Ritz solution technique are employed for the modeling of the structure. Under this theoretical framework, the effect of the cutout is taken into account by subtracting the energies of the cutout domains from the total energies of the whole plate with arbitrary boundary conditions. The displacement of the rectangular plate with nonuniform physic parameters is expressed as the combination of a two-dimensional trigonometric cosine series and supplementary terms introduced to ensure the uniform convergence of the solution over the entire solution domain including the cutouts boundary. The effectiveness and reliability of the eigenmodes of the rectangular plate with cutouts are checked against the results obtained by the finite element method (FEM). The cutout number, position, and size are varied to illustrate the effect of the cutouts on the vibration characteristics of the rectangular plate with cutouts.
\end{abstract}

\section{Introduction}

Rectangular plates with holes are used in many types of mechanical structures to lighten the weight of a structure, to obtain the convenient connection of structures, or to change the resonant frequency of a structure. Thus, knowledge of the vibration characteristics of rectangular plate with cutouts is taking important role in the design of ship structure, aircraft cabin, and mechanical device. As a result of the introduction of cutouts, the solution of vibration characteristics of the plate would become complex.

The position and number for cutouts in the flexible plate have great influence on the vibration characteristics of structure [1-7]. The rigidity of the flexible plate with cutouts is depended mostly upon the number of cutouts $[1,2]$. Avalos et al. [1] have investigated the transverse vibration of rectangular plate with a rectangular cutout. Based on the work, they [2] have also investigated the transverse vibration of the rectangular plate with two cutouts. The positions of the cutouts have some influence on the mode shape of the flexible plate with cutouts. Most previous investigations have been confined to discuss the influence of cutout size and boundary conditions [3-8], in which the positions of the cutouts are located in the center of the flexible plate. Takahashi [3] investigated the natural frequencies of rectangular plate with a central circular hole by the Ritz method and bar-solutions. Paramasivam [4] investigated the effect of rectangular cutout size on the fundamental frequencies for different types of boundary conditions by extending the grid framework model. Ali and Atwal [5] predicted the natural frequencies of rectangular plates with various sizes of rectangular cutouts by using the Rayleigh's method. However, the accuracy of mode results decreased with increase in the mode number and also with increase in the size of the cutout. The large amplitude flexural vibration of rectangular plates with rectangular cutouts is investigated by Reddy [6] through using a shear deformable element. The numerical results of orthotropic plates and composite plates demonstrated the effect of cutouts and boundary conditions on the natural frequencies. Lam [7] applied the modified Rayleigh-Ritz 
method to solve the vibration problems of a plate with cutouts and plates with internal nonhomogeneity. In his study, the plate domain is divided into smaller rectangular segments by the openings located centrally.

Various methods [9-12] have been presented for the vibration of plates with cutouts. The prediction of the corresponding eigenmodes has been studied in the early time. Aksu and Ali [11] have taken use of a two-dimensional finite difference scheme to predict the natural frequencies and modal behavior of plates with rectangular cutouts. In their work, the influence of the cutouts on the natural frequencies and mode shapes has also been considered. Generally, the effect of the hole is taken into account by subtracting the energies of the hole domain from the total energies of the whole plate. In doing so, the integrals of the functions consisting of the admissible functions based on the plate domain over hole domain would lead to complex numerical computations. To resolve the solution problem of the plate with a rectangular hole or a circular hole, Kwak and Han [12] proposed a kind of new method called Independent Coordinate Coupling Method (ICCM), in which the energies corresponding to the rectangular plate domain and the hole domain were derived independently and the two independent coordinates were coupled by imposing kinematic relations. The Rayleigh-Ritz method [13-18] is one of the most popular methods used in the free vibration analysis of individual plates. Mundkur [13] proposed an approximate method for the vibration of square plates with square cutouts, which is based on boundary characteristic orthogonal polynomial functions in the Rayleigh-Ritz method. The double Fourier series and Rayleigh-Ritz method were used by Laura [15] to determine the fundamental frequency for the rectangular plate with a hole, in which the rectangular plate with a hole is considered as a nonhomogeneous structure. In the above methods proposed, the plates with cutouts are usually treated as a kind of plates with nonuniform thickness. Huang and Sakiyama [17] adopted discrete Green function to analyze the free vibration of rectangular plates with an arbitrarily located hole of different shapes.

The investigations on the influence of opening positions are mostly concerned on the cutouts located in the center of the flexible plate. Due to the limitation of calculation difficulty, the cutout number investigated is relatively less. In the existed methods, the cutouts in the plate are usually considered structures with zero thickness. In order to really consider the physical characteristics of cutouts, the influence of cutout number and position on the vibration characteristic of the plate would be considered from the aspect of energy. In this paper, the use of energy principles in conjunction with Rayleigh-Ritz solution technique is developed as a method for the determination of vibration characteristics of rectangular plates with cutouts. The effect of the cutout with arbitrary boundary conditions is taken into account by subtracting the energies of the cutout domains from the total energies of the whole plate. The improved twodimensional Fourier series is introduced to represent the displacement of the plate with nonuniform characteristic parameters. The energy function of the whole structure is minimized with respect to the unknown Fourier coefficients and the eigenmodes are obtained from the solution of a linear eigenvalue problem. Natural frequencies and corresponding mode shapes of rectangular plates with cutouts have been predicted and numerically verified. The number, position, and size for the cutouts considered in this paper are variable. The influence of the boundary conditions, the cutout number, and the cutout position would be investigated in numerical results.

\section{Theoretical Formulations}

The flexible plate with cutouts shown in Figure 1 has general boundary conditions, which are simulated by assigning the translational and rotational restraining springs with proper stiffness constants. The length and width of the flexible plate with cutouts are, respectively, $L_{x}$ and $L_{y}$. The number and position of the cutouts in the flexible plate are variable. The dimensions of the cutouts are the same, whose length and width are $\left(X_{d i}-X_{c i}\right)$ and $\left(Y_{d i}-Y_{c i}\right)$, respectively. Here $i$ denotes the sequence of cutouts in the plate. The modal characteristics for the plate with cutouts are different from ones for the complete rectangular plate.

Both the governing equations and boundary conditions for the flexible plate with cutouts are, respectively, given as follows [19]:

$$
D \nabla^{4} w(x, y)-\rho h \omega^{2} w(x, y)=0 .
$$

On $x=0$,

$$
\begin{aligned}
k_{x 0} w(x, y) & =-D\left[\frac{\partial^{3} w}{\partial x^{3}}+(2-\mu) \frac{\partial^{3} w}{\partial x \partial y^{2}}\right] \\
K_{x 0} \frac{\partial w}{\partial x} & =D\left(\frac{\partial^{2} w}{\partial x^{2}}+\mu \frac{\partial^{2} w}{\partial y^{2}}\right) .
\end{aligned}
$$

On $x=X_{c i}$,

$$
\begin{aligned}
k_{x X c i} w(x, y) & =-D\left[\frac{\partial^{3} w}{\partial x^{3}}+(2-\mu) \frac{\partial^{3} w}{\partial x \partial y^{2}}\right], \\
K_{x X c i} \frac{\partial w}{\partial x} & =D\left(\frac{\partial^{2} w}{\partial x^{2}}+\mu \frac{\partial^{2} w}{\partial y^{2}}\right) .
\end{aligned}
$$

On $x=L_{x}$,

$$
\begin{aligned}
k_{x L_{x}} w(x, y) & =D\left[\frac{\partial^{3} w}{\partial x^{3}}+(2-\mu) \frac{\partial^{3} w}{\partial x \partial y^{2}}\right], \\
K_{x L_{x}} \frac{\partial w}{\partial x} & =-D\left(\frac{\partial^{2} w}{\partial x^{2}}+\mu \frac{\partial^{2} w}{\partial y^{2}}\right) .
\end{aligned}
$$

On $x=X_{d i}$,

$$
\begin{gathered}
k_{x X d i} w(x, y)=D\left[\frac{\partial^{3} w}{\partial x^{3}}+(2-\mu) \frac{\partial^{3} w}{\partial x \partial y^{2}}\right] \\
K_{x X d i} \frac{\partial w}{\partial x}=-D\left(\frac{\partial^{2} w}{\partial x^{2}}+\mu \frac{\partial^{2} w}{\partial y^{2}}\right) .
\end{gathered}
$$




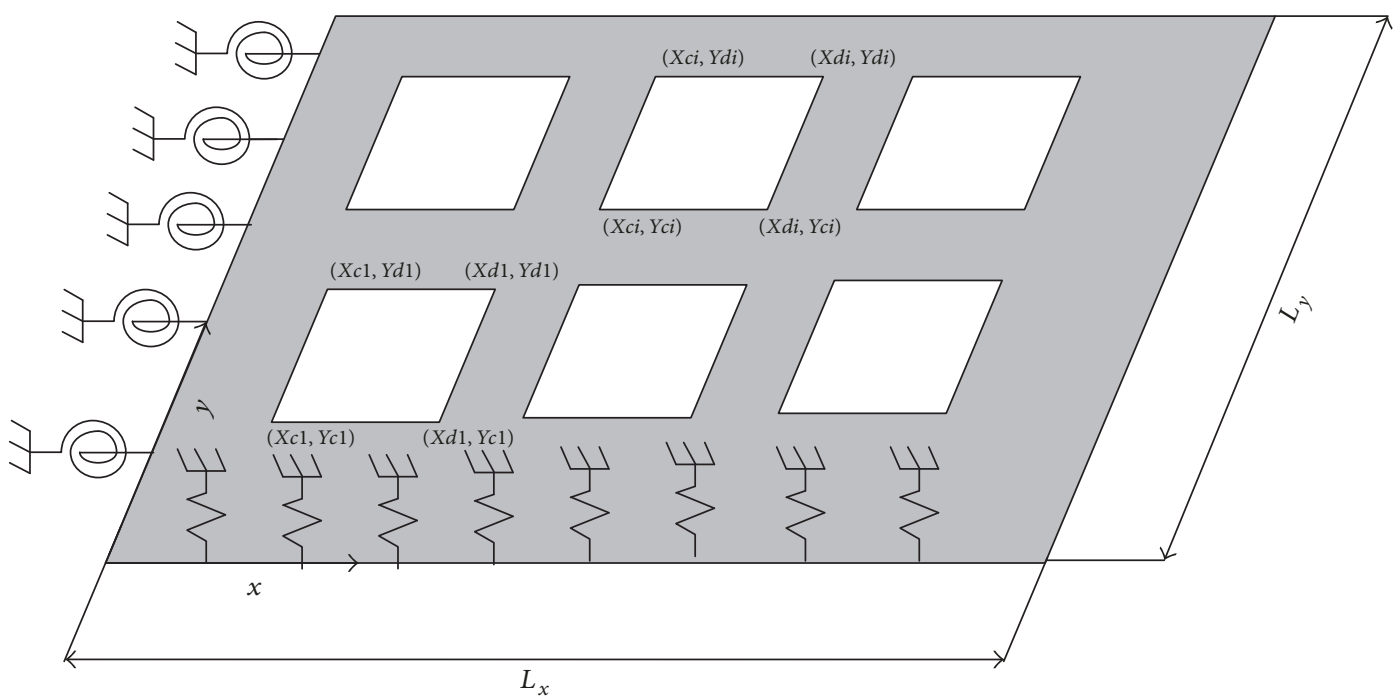

FIGURE 1: The flexible plate with cutouts.

On $y=0$

$$
\begin{aligned}
k_{y 0} w(x, y) & =-D\left[\frac{\partial^{3} w}{\partial y^{3}}+(2-\mu) \frac{\partial^{3} w}{\partial x^{2} \partial y}\right] \\
K_{y 0} \frac{\partial w}{\partial y} & =D\left(\frac{\partial^{2} w}{\partial y^{2}}+\mu \frac{\partial^{2} w}{\partial x^{2}}\right) .
\end{aligned}
$$

On $y=Y_{c i}$,

$$
\begin{aligned}
k_{y Y c i} w(x, y) & =-D\left[\frac{\partial^{3} w}{\partial y^{3}}+(2-\mu) \frac{\partial^{3} w}{\partial x^{2} \partial y}\right] \\
K_{y Y c i} \frac{\partial w}{\partial y} & =D\left(\frac{\partial^{2} w}{\partial y^{2}}+\mu \frac{\partial^{2} w}{\partial x^{2}}\right) .
\end{aligned}
$$

On $y=L_{y}$,

$$
\begin{aligned}
k_{y L_{y}} w(x, y) & =D\left[\frac{\partial^{3} w}{\partial y^{3}}+(2-\mu) \frac{\partial^{3} w}{\partial x^{2} \partial y}\right] \\
K_{y L_{y}} \frac{\partial w}{\partial y} & =-D\left(\frac{\partial^{2} w}{\partial y^{2}}+\mu \frac{\partial^{2} w}{\partial x^{2}}\right) .
\end{aligned}
$$

On $y=Y_{d i}$,

$$
\begin{aligned}
k_{y Y d i} w(x, y) & =D\left[\frac{\partial^{3} w}{\partial y^{3}}+(2-\mu) \frac{\partial^{3} w}{\partial x^{2} \partial y}\right], \\
K_{y Y d i} \frac{\partial w}{\partial y} & =-D\left(\frac{\partial^{2} w}{\partial y^{2}}+\mu \frac{\partial^{2} w}{\partial x^{2}}\right),
\end{aligned}
$$

where $D$ and $\mu$ are, respectively, the bending stiffness and the Poisson's ratio; $\rho$ and $h$ are, respectively, the mass density and thickness of the flexible panel; $k_{x 0}, k_{x L x}, k_{y 0}, k_{y L y}, k_{x X c i}$, $k_{x X d i}, k_{y Y c i}$, and $k_{y Y d i}$ denote the edge stiffness of the translational restraining springs; $K_{x 0}, K_{x L x}, K_{y 0}, K_{y L y}, K_{x X c i}$, $K_{x X d i}, K_{y Y c i}$, and $K_{y Y d i}$ denote the edge stiffness of the rotational restraining springs. The boundary conditions can be represented by both the translational and rotational springs along each edge and all classical homogeneous boundary conditions can be easily derived by simply setting each of the spring stiffness to be an extremely large or small number. The displacement expression $w(x, y)$ in the above equations is given as [19]

$$
\begin{aligned}
& W(x, y)=\sum_{m=0}^{\infty} \sum_{n=0}^{\infty} A_{m n} \cos \lambda_{L_{x} m} x \cos \lambda_{L_{y} n} y \\
& +\sum_{m=0}^{\infty}[\underbrace{a_{m} \zeta_{1 L_{y}}(y)+c_{m} \zeta_{3 L_{y}}(y)}_{y=0} \\
& +\underbrace{b_{m} \zeta_{2 L_{y}}(y)+d_{m} \zeta_{4 L_{y}}(y)}_{y=L_{y}}] \cos \lambda_{L_{x} m} x \\
& +\sum_{n=0}^{\sum_{x}^{\infty}[\underbrace{e_{1 n} \zeta_{1 L_{x}}(x)+g_{1 n} \zeta_{3 L_{x}}}_{x=0}(x)} \\
& +\underbrace{f_{1 n} \zeta_{2 L_{x}}(x)+h_{1 n} \zeta_{4 L_{x}}(x)}_{x=L_{x}}] \cos \lambda_{L_{y} n} y,
\end{aligned}
$$

where $\lambda_{L_{x} m}=m \pi / L_{x}, \lambda_{L_{y} n}=n \pi / L_{y}$, and

$$
\begin{aligned}
& \zeta_{1 L_{x}}(x)=\frac{9 L_{x}}{4 \pi} \sin \left(\frac{\pi x}{2 L_{x}}\right)-\frac{L_{x}}{12 \pi} \sin \left(\frac{3 \pi x}{2 L_{x}}\right), \\
& \zeta_{2 L_{x}}(x)=-\frac{9 L_{x}}{4 \pi} \cos \left(\frac{\pi x}{2 L_{x}}\right)-\frac{L_{x}}{12 \pi} \cos \left(\frac{3 \pi x}{2 L_{x}}\right),
\end{aligned}
$$




$$
\begin{aligned}
& \zeta_{3 L_{x}}(x)=\frac{L_{x}^{3}}{\pi^{3}} \sin \left(\frac{\pi x}{2 L_{x}}\right)-\frac{L_{x}^{3}}{3 \pi^{3}} \sin \left(\frac{3 \pi x}{2 L_{x}}\right), \\
& \zeta_{4 L_{x}}(x)=-\frac{L_{x}^{3}}{\pi^{3}} \cos \left(\frac{\pi x}{2 L_{x}}\right)-\frac{L_{x}^{3}}{3 \pi^{3}} \cos \left(\frac{3 \pi x}{2 L_{x}}\right) .
\end{aligned}
$$

According to the expressions of admissible displacement functions given above, the first derivation of the admissible displacement could be derived as follows:

$$
\begin{aligned}
\zeta_{1 L_{x}}^{\prime}(x)= & \frac{9 L_{x}}{4 \pi} \frac{\pi}{2 L_{x}} \cos \left(\frac{\pi x}{2 L_{x}}\right) \\
& -\frac{L_{x}}{12 \pi} \frac{3 \pi}{2 L_{x}} \cos \left(\frac{3 \pi x}{2 L_{x}}\right), \\
\zeta_{2 L_{x}}^{\prime}(x)= & \frac{9 L_{x}}{4 \pi} \frac{\pi}{2 L_{x}} \sin \left(\frac{\pi x}{2 L_{x}}\right) \\
& +\frac{L_{x}}{12 \pi} \frac{3 \pi}{2 L_{x}} \sin \left(\frac{3 \pi x}{2 L_{x}}\right), \\
\zeta_{3 L_{x}}^{\prime}(x)= & \frac{L_{x}^{3}}{\pi^{3}} \frac{\pi}{2 L_{x}} \cos \left(\frac{\pi x}{2 L_{x}}\right) \\
& -\frac{L_{x}^{3}}{3 \pi^{3}} \frac{3 \pi}{2 L_{x}} \cos \left(\frac{3 \pi x}{2 L_{x}}\right), \\
\zeta_{4 L_{x}}^{\prime}(x)= & \frac{L_{x}^{3}}{\pi^{3}} \frac{\pi}{2 L_{x}} \sin \left(\frac{\pi x}{2 L_{x}}\right) \\
& \frac{L_{x}^{3}}{3 \pi^{3}} \frac{3 \pi}{2 L_{x}} \sin \left(\frac{3 \pi x}{2 L_{x}}\right) .
\end{aligned}
$$

The third derivation of admissible displacement could be obtained from the first derivations:

$$
\begin{aligned}
\zeta_{1 L_{x}}^{\prime \prime \prime}(x)= & -\frac{9 L_{x}}{4 \pi} \frac{\pi}{2 L_{x}} \frac{\pi}{2 L_{x}} \frac{\pi}{2 L_{x}} \cos \left(\frac{\pi x}{2 L_{x}}\right) \\
& +\frac{L_{x}}{12 \pi} \frac{3 \pi}{2 L_{x}} \frac{3 \pi}{2 L_{x}} \frac{3 \pi}{2 L_{x}} \cos \left(\frac{3 \pi x}{2 L_{x}}\right), \\
\zeta_{2 L_{x}}^{\prime \prime \prime}(x)= & -\frac{9 L_{x}}{4 \pi} \frac{\pi}{2 L_{x}} \frac{\pi}{2 L_{x}} \frac{\pi}{2 L_{x}} \sin \left(\frac{\pi x}{2 L_{x}}\right) \\
& -\frac{L_{x}}{12 \pi} \frac{3 \pi}{2 L_{x}} \frac{3 \pi}{2 L_{x}} \frac{3 \pi}{2 L_{x}} \sin \left(\frac{3 \pi x}{2 L_{x}}\right), \\
\zeta_{3 L_{x}}^{\prime \prime \prime}(x)= & -\frac{L_{x}^{3}}{\pi^{3}} \frac{\pi}{2 L_{x}} \frac{\pi}{2 L_{x}} \frac{\pi}{2 L_{x}} \cos \left(\frac{\pi x}{2 L_{x}}\right) \\
& +\frac{L_{x}^{3}}{3 \pi^{3}} \frac{3 \pi}{2 L_{x}} \frac{3 \pi}{2 L_{x}} \frac{3 \pi}{2 L_{x}} \cos \left(\frac{3 \pi x}{2 L_{x}}\right), \\
\zeta_{4 L_{x}}^{\prime \prime \prime}(x)= & -\frac{L_{x}^{3}}{\pi^{3}} \frac{\pi}{2 L_{x}} \frac{\pi}{2 L_{x}} \frac{\pi}{2 L_{x}} \sin \left(\frac{\pi x}{2 L_{x}}\right) \\
& \frac{L_{x}^{3}}{2 L_{x}} \frac{3 \pi}{2 L_{x}} \frac{3 \pi}{2 L_{x}} \sin \left(\frac{3 \pi x}{2 L_{x}}\right) .
\end{aligned}
$$

The characteristic for admissible functions at $x=0$ and $x=L_{x}$ could be obtained from the expressions of admissible functions, which is

$$
\zeta_{1 L_{x}}^{\prime}(0)=\zeta_{3 L_{x}}^{\prime \prime \prime}(0)=\zeta_{2 L_{x}}^{\prime}\left(L_{x}\right)=\zeta_{4 L_{x}}^{\prime \prime \prime}\left(L_{x}\right)=1 .
$$

If the dynamic characteristic of flexible plate with cutouts is obtained from the above governing equations and boundary conditions, the solution process would become more and more complex due to the increase of cutout number. In this paper, the energy principle is adopted to carry out the modal analysis of flexible plate with cutouts, which is equivalent to the solution obtained from the method given above.

The energy of the flexible plate with cutouts could be obtained by deleting the energy of the flexible plate occupying the cutout from the energy for the flexible plate without cutouts. The boundary conditions of the cutouts are depended on the location of the cutouts. If the cutout is in the flexible plate, the boundary conditions for the cutout are free. If the edge of cutout is laid on the edge of flexible plate, the boundary conditions for the edge of the cutout are the same with those for the edge of the flexible plate. Thus, the Lagrange function for the flexible plate with $n$ cutouts is given as

$$
\begin{aligned}
L= & \left(V-V_{1}-V_{2}-\cdots-V_{i}-\cdots-V_{n}\right) \\
& -\left(T-T_{1}-T_{2}-\cdots-T_{i}-\cdots-T_{n}\right),
\end{aligned}
$$

where $V$ and $T$ denote the potential and kinetic energy of flexible plate without cutouts, respectively; $V_{i}$ and $T_{i}$ denote the potential and kinetic energy of the flexible plate occupying the $i$ th cutout, respectively.

The strain and kinetic energy of the transverse vibration of flexible plate without cutouts can be obtained as [19]

$$
\begin{aligned}
V & =\frac{D}{2} \int_{0}^{L_{x}} \int_{0}^{L_{y}}\left\{\left(\frac{\partial^{2} w}{\partial x^{2}}\right)^{2}+\left(\frac{\partial^{2} w}{\partial y^{2}}\right)^{2}+2 \mu \frac{\partial^{2} w}{\partial x^{2}} \frac{\partial^{2} w}{\partial y^{2}}\right. \\
& \left.+2(1-\mu)\left(\frac{\partial^{2} w}{\partial x \partial y}\right)^{2}\right\} \mathrm{d} x \mathrm{~d} y+\frac{1}{2} \\
& \cdot \int_{0}^{L_{y}}\left[k_{x 0} w^{2}+K_{x 0}\left(\frac{\partial w}{\partial x}\right)^{2}\right]_{x=0} \mathrm{~d} y+\frac{1}{2} \\
& \cdot \int_{0}^{L_{y}}\left[k_{x L_{x}} w^{2}+K_{x L_{x}}\left(\frac{\partial w}{\partial x}\right)^{2}\right]_{x=L_{x}} \mathrm{~d} y+\frac{1}{2} \\
& \cdot \int_{0}^{L_{x}}\left[k_{y 0} w^{2}+K_{y 0}\left(\frac{\partial w}{\partial y}\right)^{2}\right]_{y=0} \mathrm{~d} x+\frac{1}{2} \\
& \cdot \int_{0}^{L_{x}}\left[k_{y L_{y}} w^{2}+K_{y L_{y}}\left(\frac{\partial w}{\partial x}\right)^{2}\right]_{y=L_{y}} \mathrm{~d} x, \\
T= & \frac{1}{2} \int_{0}^{L_{y}} \int_{0}^{L_{x}} \rho h\left(\frac{\partial w}{\partial t}\right)^{2} \mathrm{~d} x \mathrm{~d} y=\frac{1}{2} \\
& \cdot \rho h \omega^{2} \int_{0}^{L_{y}} \int_{0}^{L_{x}} w^{2} \mathrm{~d} x \mathrm{~d} y .
\end{aligned}
$$


The strain and kinetic energy stored in the flexible plate occupying the $i$ th cutout are expressed as

$$
\begin{aligned}
V_{i} & =\frac{D}{2} \int_{X c i}^{X d i} \int_{Y c i}^{Y d i}\left\{\left(\frac{\partial^{2} w}{\partial x^{2}}\right)^{2}+\left(\frac{\partial^{2} w}{\partial y^{2}}\right)^{2}\right. \\
& \left.+2 \mu \frac{\partial^{2} w}{\partial x^{2}} \frac{\partial^{2} w}{\partial y^{2}}+2(1-\mu)\left(\frac{\partial^{2} w}{\partial x \partial y}\right)^{2}\right\} \mathrm{d} x \mathrm{~d} y \\
& +\frac{1}{2} \int_{Y c i}^{Y d i}\left[k_{x X c i} w^{2}+K_{x X c i}\left(\frac{\partial w}{\partial x}\right)^{2}\right]_{X=X c i} \mathrm{~d} y+\frac{1}{2} \\
& \cdot \int_{Y c i}^{Y d i}\left[k_{x X d i} w^{2}+K_{x X d i}\left(\frac{\partial w}{\partial x}\right)^{2}\right]_{x=X d i} \mathrm{~d} y+\frac{1}{2} \\
& \cdot \int_{X c i}^{X d i}\left[k_{y Y c i} w^{2}+K_{y Y c i}\left(\frac{\partial w}{\partial y}\right)^{2}\right]_{y=Y c i} \mathrm{~d} x+\frac{1}{2} \\
& \cdot \int_{Y c i}^{Y d i}\left[k_{y Y d i} w^{2}+K_{y Y d i}\left(\frac{\partial w}{\partial y}\right)^{2}\right]_{y=Y d i} \mathrm{~d} x, \\
& \cdot \rho h \omega^{2} \int_{X c i}^{X d i} \int_{Y c i}^{Y d i} w^{2} \mathrm{~d} x \mathrm{~d} y . \\
T_{i} & \frac{1}{2} \int_{X c i}^{X d i} \int_{Y c i}^{Y d i} \rho h\left(\frac{\partial w}{\partial t}\right)^{2} \mathrm{~d} x \mathrm{~d} y=\frac{1}{2}
\end{aligned}
$$

By substituting (16) and (17) into (15) and applying the Rayleigh-Ritz procedure against each of the unknown Fourier series coefficients, the linear system in the matrix form may be described as

$$
\left(\mathbf{K}-\left(\sum_{i=1}^{n} \mathbf{K}_{i}\right)\right)-\omega^{2}\left(\mathbf{M}-\left(\sum_{i=1}^{n} \mathbf{M}_{i}\right)\right) \mathbf{E}=\mathbf{0}
$$

where

$$
\begin{aligned}
\mathbf{K} & =\left[\begin{array}{cccccc}
\mathbf{K}_{1-1} & \mathbf{K}_{1-2} & \mathbf{K}_{1-3} & \mathbf{K}_{1-4} & \cdots & \mathbf{K}_{1-9} \\
\mathbf{K}_{2-1} & \mathbf{K}_{2-2} & \mathbf{K}_{2-3} & \mathbf{K}_{2-4} & \cdots & \mathbf{K}_{2-9} \\
\mathbf{K}_{3-1} & \mathbf{K}_{3-2} & \mathbf{K}_{3-3} & \mathbf{K}_{3-4} & \cdots & \mathbf{K}_{3-9} \\
\mathbf{K}_{4-1} & \mathbf{K}_{4-2} & \mathbf{K}_{4-3} & \mathbf{K}_{4-4} & \cdots & \mathbf{K}_{4-9} \\
\vdots & \vdots & \vdots & \vdots & \ddots & \vdots \\
\mathbf{K}_{9-1} & \mathbf{K}_{9-2} & \mathbf{K}_{9-3} & \mathbf{K}_{9-4} & \cdots & \mathbf{K}_{9-9}
\end{array}\right], \\
\mathbf{M} & =\left[\begin{array}{cccccc}
\mathbf{M}_{1-1} & \mathbf{M}_{1-2} & \mathbf{M}_{1-3} & \mathbf{M}_{1-4} & \cdots & \mathbf{M}_{1-9} \\
\mathbf{M}_{2-1} & \mathbf{M}_{2-2} & \mathbf{M}_{2-3} & \mathbf{M}_{2-4} & \cdots & \mathbf{M}_{2-9} \\
\mathbf{M}_{3-1} & \mathbf{M}_{3-2} & \mathbf{M}_{3-3} & \mathbf{M}_{3-4} & \cdots & \mathbf{M}_{3-9} \\
\mathbf{M}_{4-1} & \mathbf{M}_{4-2} & \mathbf{M}_{4-3} & \mathbf{M}_{4-4} & \cdots & \mathbf{M}_{4-9} \\
\vdots & \vdots & \vdots & \vdots & \ddots & \vdots \\
\mathbf{M}_{9-1} & \mathbf{M}_{9-2} & \mathbf{M}_{9-3} & \mathbf{M}_{9-4} & \cdots & \mathbf{M}_{9-9}
\end{array}\right],
\end{aligned}
$$

$$
\begin{aligned}
& \mathbf{K}_{i}=\left[\begin{array}{llllll}
\mathbf{K}_{1-1}^{i} & \mathbf{K}_{1-2}^{i} & \mathbf{K}_{1-3}^{i} & \mathbf{K}_{1-4}^{i} & \cdots & \mathbf{K}_{1-9}^{i} \\
\mathbf{K}_{2-1}^{i} & \mathbf{K}_{2-2}^{i} & \mathbf{K}_{2-3}^{i} & \mathbf{K}_{2-4}^{i} & \cdots & \mathbf{K}_{2-9}^{i} \\
\mathbf{K}_{3-1}^{i} & \mathbf{K}_{3-2}^{i} & \mathbf{K}_{3-3}^{i} & \mathbf{K}_{3-4}^{i} & \cdots & \mathbf{K}_{3-9}^{i} \\
\mathbf{K}_{4-1}^{i} & \mathbf{K}_{4-2}^{i} & \mathbf{K}_{4-3}^{i} & \mathbf{K}_{4-4}^{i} & \cdots & \mathbf{K}_{4-9}^{i} \\
\vdots & \vdots & \vdots & \vdots & \ddots & \vdots \\
\mathbf{K}_{9-1}^{i} & \mathbf{K}_{9-2}^{i} & \mathbf{K}_{9-3}^{i} & \mathbf{K}_{9-4}^{i} & \cdots & \mathbf{K}_{9-9}^{i}
\end{array}\right], \\
& \mathbf{M}_{i}=\left[\begin{array}{cccccc}
\mathbf{M}_{1-1}^{i} & \mathbf{M}_{1-2}^{i} & \mathbf{M}_{1-3}^{i} & \mathbf{M}_{1-4}^{i} & \cdots & \mathbf{M}_{1-9}^{i} \\
\mathbf{M}_{2-1}^{i} & \mathbf{M}_{2-2}^{i} & \mathbf{M}_{2-3}^{i} & \mathbf{M}_{2-4}^{i} & \cdots & \mathbf{M}_{2-9}^{i} \\
\mathbf{M}_{3-1}^{i} & \mathbf{M}_{3-2}^{i} & \mathbf{M}_{3-3}^{i} & \mathbf{M}_{3-4}^{i} & \cdots & \mathbf{M}_{3-9}^{i} \\
\mathbf{M}_{4-1}^{i} & \mathbf{M}_{4-2}^{i} & \mathbf{M}_{4-3}^{i} & \mathbf{M}_{4-4}^{i} & \cdots & \mathbf{M}_{4-9}^{i} \\
\vdots & \vdots & \vdots & \vdots & \ddots & \vdots \\
\mathbf{M}_{9-1}^{i} & \mathbf{M}_{9-2}^{i} & \mathbf{M}_{9-3}^{i} & \mathbf{M}_{9-4}^{i} & \cdots & \mathbf{M}_{9-9}^{i}
\end{array}\right], \\
& \mathbf{E}=\left\{A_{00}^{p}, A_{01}^{p}, \ldots, A_{m 0}^{p}, A_{m 1}^{p}, \ldots, A_{m n}^{p}, \ldots, A_{M N}^{p}, a_{0}^{p}, \ldots,\right. \\
& a_{M}^{p}, b_{0}^{p}, \ldots, b_{M}^{p}, c_{0}^{p}, \ldots, c_{M}^{p}, d_{0}^{p}, \ldots, d_{M}^{p}, e_{0}^{p}, \ldots, e_{N}^{p}, f_{0}^{p}, \ldots, \\
& \left.f_{N}^{p}, g_{0}^{p}, \ldots, g_{N}^{p}, h_{0}^{p}, \ldots, h_{N}^{p}\right\}^{T} \text {, }
\end{aligned}
$$

where $\boldsymbol{K}$ and $\boldsymbol{M}$ are stiffness and mass matrices of the flexible plate without cutouts, respectively; $\boldsymbol{K}_{i}$ and $\mathbf{M}_{i}$ are stiffness and mass matrices of the flexible plate occupying the $i$ th cutout, respectively. The expressions of these matrices are given in the Appendix. For numerical calculations, all the Fourier series are truncated to $m_{p}=M_{p}, n_{p}=N_{p}$. The accuracy of the calculation results can be improved by the increase of the Fourier truncated series. The coefficient vectors $\boldsymbol{E}$ can be obtained by solving (18). Knowing the expansion coefficients $\boldsymbol{E}$, the displacement of the flexible panel with cutouts can be easily obtained from (10). The transient analysis of the mathematical model could also be calculated by revising the zero vector on the right of (18) into the loading vector.

\section{Results and Discussions}

3.1. Convergence and Validation. The convergence study of the present method is conducted with a flexible plate with one cutout for different boundary conditions. The length $\left(L_{x}\right)$, width $\left(L_{y}\right)$, and thickness $(h)$ for the flexible plate with cutouts are $L_{x}=L_{y}=2 \mathrm{~m}$ and $h=0.003 \mathrm{~m}$, respectively. In the analytical investigation, Young's modulus, Poisson's ratio, and mass density are assumed to be $E=216 \mathrm{GPa}$, $v=0.3$, and $\rho=7900 \mathrm{~kg} / \mathrm{m}^{3}$, respectively. The first six natural frequencies of square plate with a square opening for different boundary conditions are listed in Table 1, in which the size and original point of the square cutout are, respectively, $1.1 \mathrm{~m}$ $\times 1.1 \mathrm{~m}$ and $(0.4 \mathrm{~m}, 0.4 \mathrm{~m})$. The alphabet $\mathrm{C}$ in the table refers to the clamped edge, $\mathrm{S}$ refers to the simply supported edge, and F means that the edge is free. As shown in Table 1, the natural frequencies for the plate with a free cutout are validated by the comparison with the simulation results and converge at $M_{p}=N_{p}=15$. The simulation results are obtained from the 
TABLE 1: Convergence of the first six natural frequencies $(\mathrm{Hz})$ of the square plate with square opening $1.1 \mathrm{~m} \times 1.1 \mathrm{~m}$ for different boundary conditions.

\begin{tabular}{|c|c|c|c|c|c|c|c|}
\hline \multirow{2}{*}{ Boundary conditions } & \multirow{2}{*}{$M_{p} \times N_{p}$} & \multicolumn{6}{|c|}{ Mode number } \\
\hline & & 1 & 2 & 3 & 4 & 5 & 6 \\
\hline \multirow{5}{*}{ CCCC-FFFF } & $12 \times 12$ & 13.2 & 15.9 & 18.7 & 22.0 & 22.6 & 29.0 \\
\hline & $14 \times 14$ & 13.2 & 15.9 & 18.7 & 21.9 & 22.5 & 28.8 \\
\hline & $15 \times 15$ & 13.2 & 15.8 & 18.7 & 21.9 & 22.4 & 28.7 \\
\hline & $16 \times 16$ & 13.2 & 15.8 & 18.7 & 21.9 & 22.4 & 28.7 \\
\hline & ANSYS & 13.1 & 15.6 & 18.7 & 21.7 & 22.0 & 28.1 \\
\hline \multirow{5}{*}{ SSSS-FFFF } & $12 \times 12$ & 4.4 & 7.8 & 7.8 & 13.6 & 14.6 & 21.6 \\
\hline & $14 \times 14$ & 4.4 & 7.7 & 7.7 & 13.6 & 14.4 & 21.5 \\
\hline & $15 \times 15$ & 4.4 & 7.7 & 7.7 & 13.5 & 14.4 & 21.5 \\
\hline & $16 \times 16$ & 4.4 & 7.7 & 7.7 & 13.5 & 14.4 & 21.5 \\
\hline & ANSYS & 4.4 & 7.6 & 7.6 & 13.5 & 13.9 & 21.1 \\
\hline
\end{tabular}

TABLE 2: Natural frequencies $(\mathrm{Hz})$ for the flexible plate with six cutouts.

\begin{tabular}{|c|c|c|c|}
\hline \multirow{2}{*}{ Mode number } & \multicolumn{2}{|c|}{ Methods } & \multirow{2}{*}{ Error } \\
\hline & Present & ANSYS & \\
\hline 1 & 7.9 & 7.8 & $1.2 \%$ \\
\hline 2 & 13.6 & 13.5 & $0.7 \%$ \\
\hline 3 & 14.6 & 14.5 & $0.6 \%$ \\
\hline 4 & 20.6 & 20.4 & $0.9 \%$ \\
\hline 5 & 20.9 & 20.5 & $1.9 \%$ \\
\hline 6 & 22.2 & 21.7 & $2.3 \%$ \\
\hline 7 & 27.6 & 26.7 & $3.3 \%$ \\
\hline 8 & 28.1 & 27.4 & $2.5 \%$ \\
\hline 9 & 28.7 & 28.1 & $2.1 \%$ \\
\hline 10 & 33.7 & 32.6 & $3.3 \%$ \\
\hline 20 & 68.0 & 67.4 & $0.8 \%$ \\
\hline 40 & 124.6 & 122.6 & $1.6 \%$ \\
\hline 80 & 238.9 & 235.7 & $1.3 \%$ \\
\hline 100 & 306.2 & 302.8 & $1.1 \%$ \\
\hline 200 & 602.8 & 601.1 & $0.2 \%$ \\
\hline
\end{tabular}

software ANSYS, whose model is constructed by setting the SHELL63 element size as $0.02 \mathrm{~m} \times 0.02 \mathrm{~m}$.

To validate the accuracy and reliability of the present method, the first 200 natural frequencies and corresponding mode shapes of clamped flexible plate with six free cutouts are compared with those obtained from the software ANSYS. The geometrical and physical parameters for the flexible plate have been given in the convergence study. The origins of the six cutouts are $(0.4 \mathrm{~m}, 0.4 \mathrm{~m}),(0.8 \mathrm{~m}, 0.4 \mathrm{~m}),(1.2 \mathrm{~m}, 0.4 \mathrm{~m})$, $(0.4 \mathrm{~m}, 1 \mathrm{~m}),(0.8 \mathrm{~m}, 1 \mathrm{~m})$, and $(1.2 \mathrm{~m}, 1 \mathrm{~m})$, respectively. Different from the above convergence study, the six cutouts with the length $l x=0.3 \mathrm{~m}$ and the width $l y=0.5 \mathrm{~m}$ are rectangular holes. The Fourier series is truncated to $M_{p}=N_{p}=15$ in this analysis. The element type and size in the simulation study are the same as the ones in the convergence study. It can be seen that the comparison between theoretical and simulation results in Table 2 and Figure 2 shows good agreement, and the difference between two results in Table 2 is less than 3.3\% for the worst case. The small differences may be caused by the division of element mesh in the numerical simulation. Compared with finite element method, mathematical model proposed in the current paper could investigate the vibration characteristics of the flexible plate in a simple and flexible way. It can be concluded from the validation that the present method is able to accurately predict the natural frequencies of flexible plate with multiple cutouts.

3.2. The Effect of Boundary Conditions and Cutouts. In this section, the influence of structural parameters on the free vibration characteristics is investigated through boundary conditions, cutout size, cutout position, and cutout number. The size and related physical conditions for the flexible plate considered here are kept the same with ones given in Section 3.1.

For the present method, the general boundary conditions of the flexible plate can be achieved by setting groups of boundary springs and assigning corresponding stiffness constants to the springs. The first six natural frequencies of 

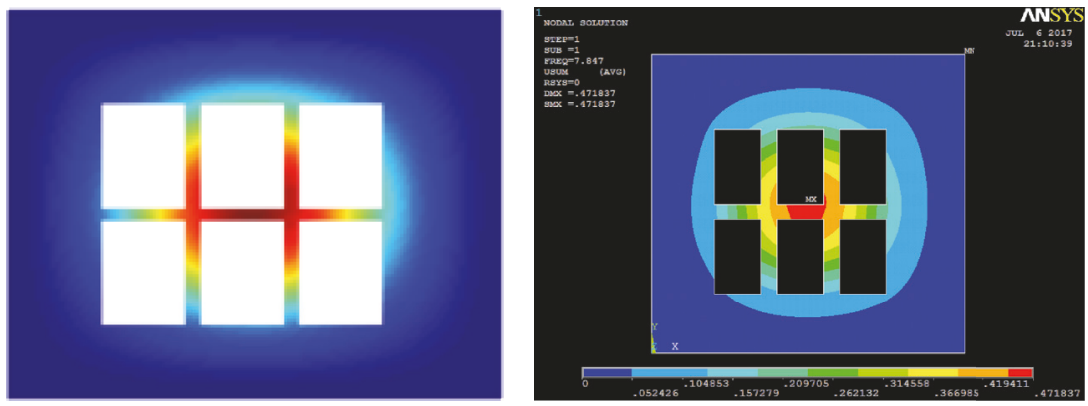

(a)
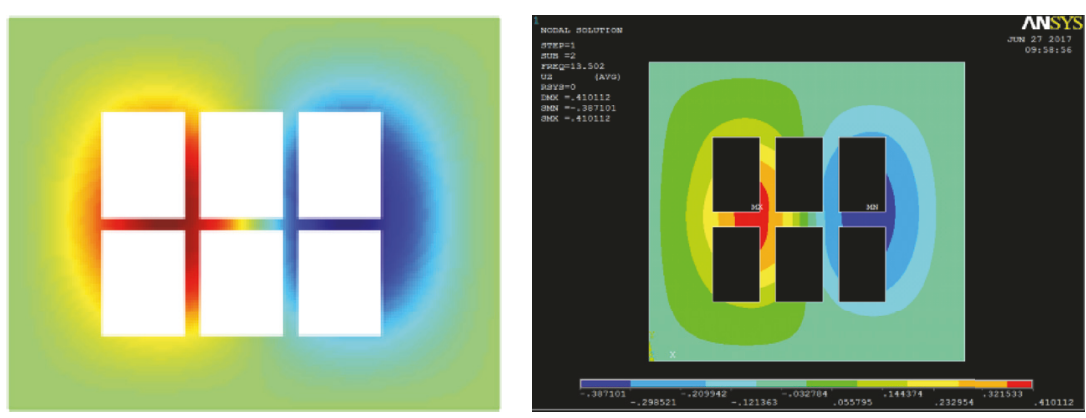

(b)
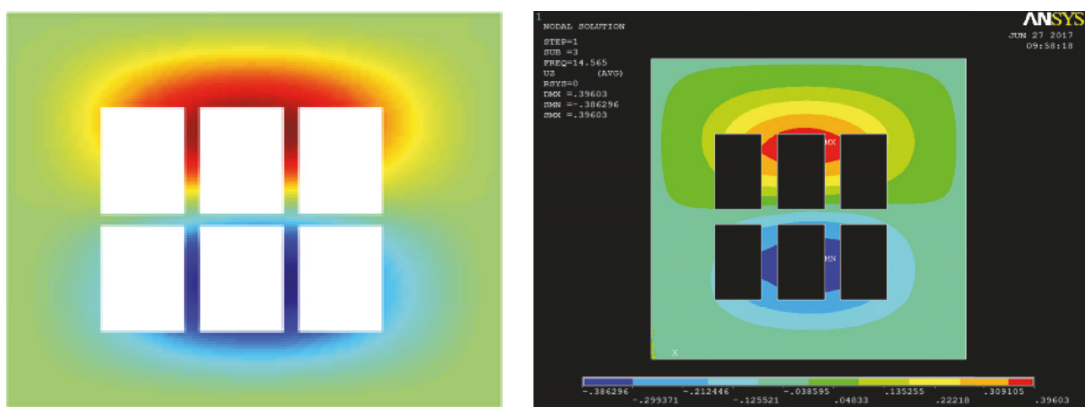

(c)
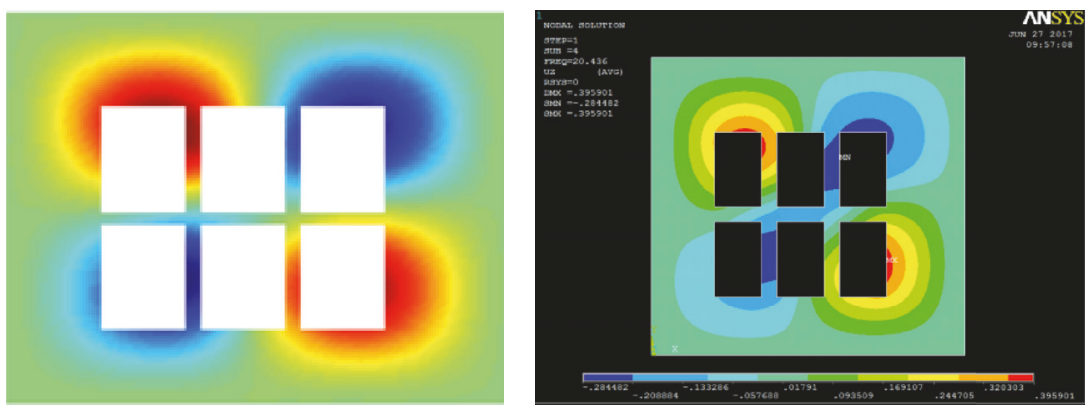

(d)
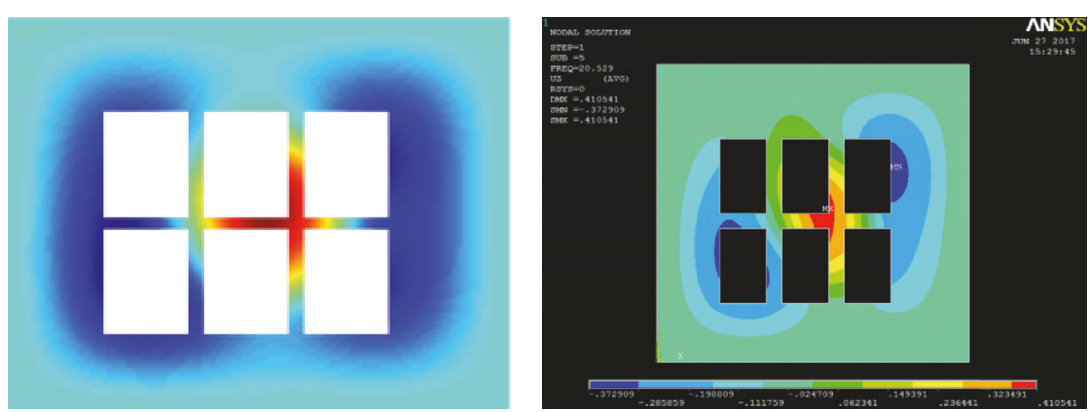

(e)

FIgUre 2: Continued. 

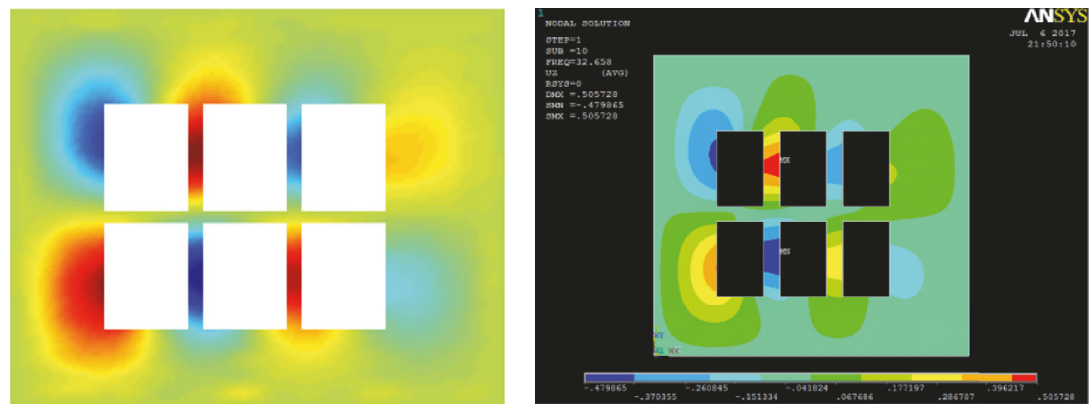

(j)
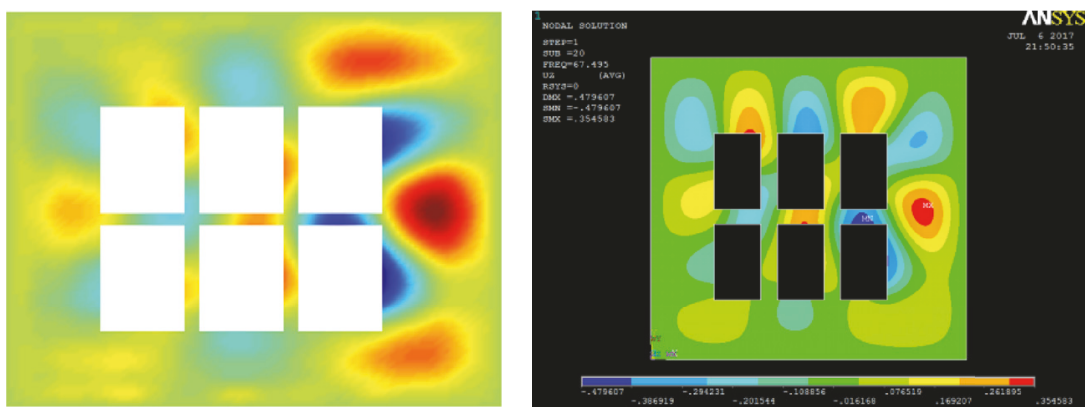

(k)
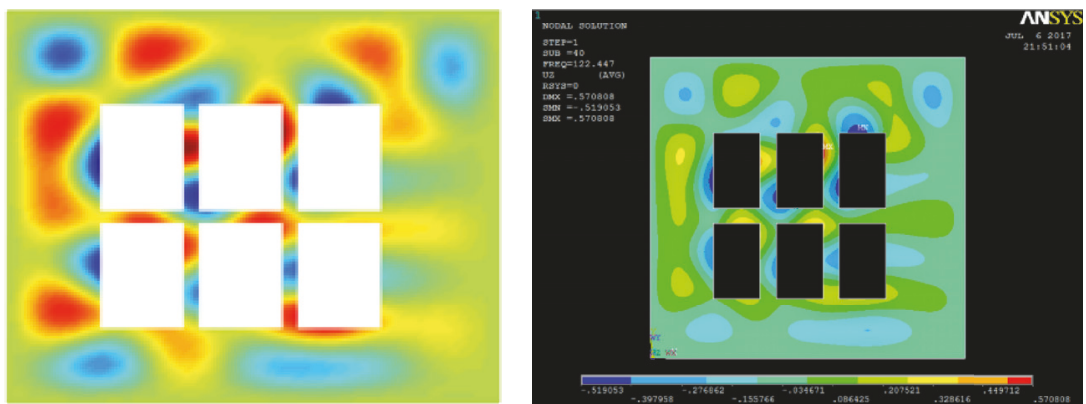

$(\mathrm{m})$
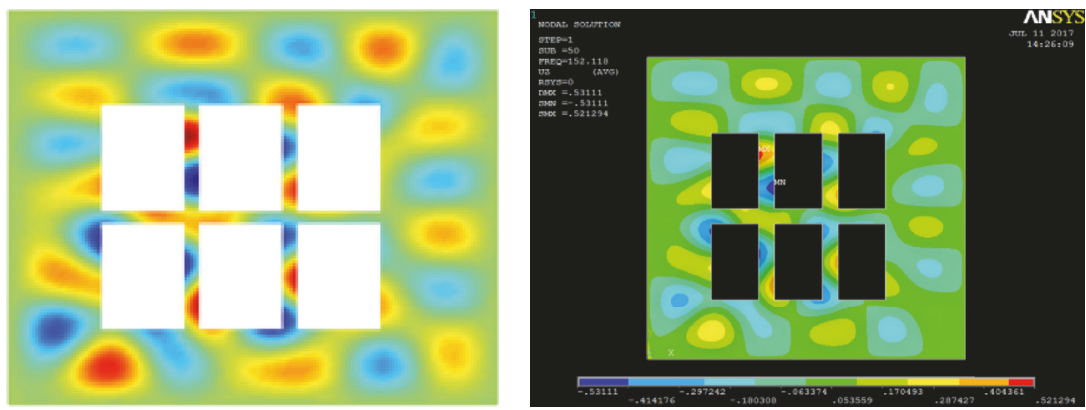

(n)

Figure 2: The mode shapes for the flexible plate with six cutouts. (a) The first mode; (b) the second mode; (c) the third mode; (d) the fourth mode; (e) the fifth mode; $(\mathrm{j})$ the tenth mode; $(\mathrm{k})$ the twentieth mode; $(\mathrm{m})$ the fortieth mode; $(\mathrm{n})$ the fiftieth mode.

the flexible plate with one free cutout for different boundary restrained stiffness are given in Figure 3. The size and origin of the cutout are, respectively, $1 \mathrm{~m} \times 1 \mathrm{~m}$ and $(0.5 \mathrm{~m}, 0.5 \mathrm{~m})$. As shown in Figure 3, the first six natural frequencies increase with the increase of the translational and rotational restraining stiffness, and the effect of the translational restraining stiffness is more remarkable. It is not difficult to find in
Figure 3(a) that the natural frequencies for the lower modes are almost liner with the translational restraining stiffness between $1 E 2$ and $1 E 6$, and the natural frequencies are more influenced by the translational spring stiffness.

In the cutout size study, the centers of the selected square cutouts are located at $(1 \mathrm{~m}, 1 \mathrm{~m})$. The first six natural frequencies of the clamped plate with one cutout for different 


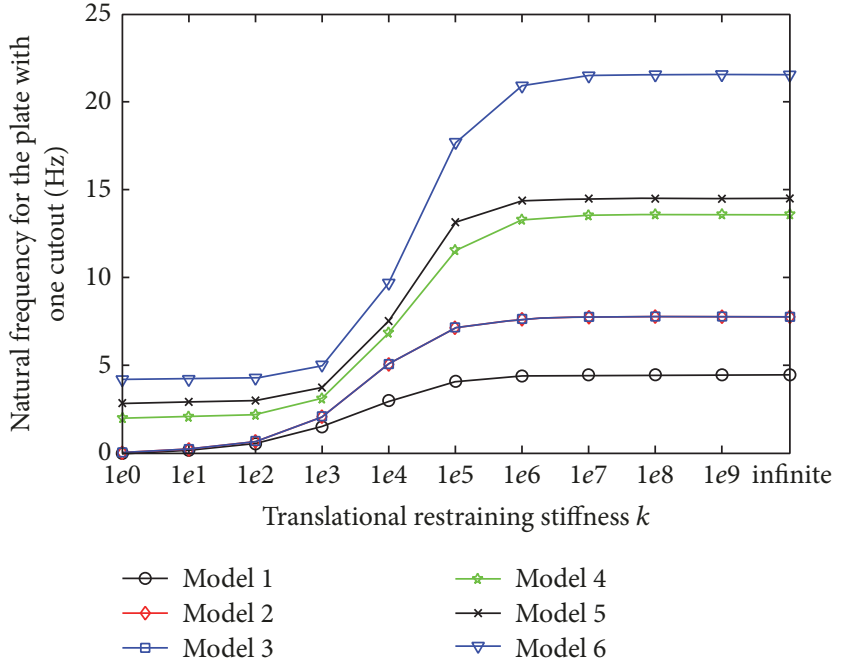

(a)

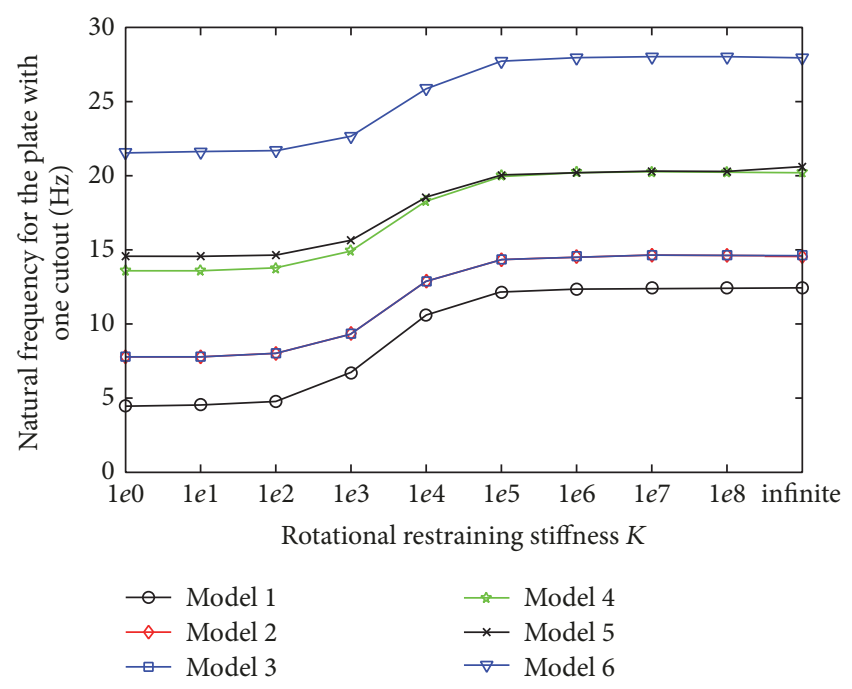

(b)

FIGURE 3: The first six natural frequencies of flexible plate with one cutout for different boundary restrained stiffness: (a) variation of the translational restraining stiffness; (b) variation of the rotational restraining stiffness.

TABLE 3: The first six natural frequencies $(\mathrm{Hz})$ for different square opening sizes.

\begin{tabular}{|c|c|c|c|c|c|c|c|}
\hline \multirow{2}{*}{ Cutout size } & \multirow{2}{*}{ Methods } & \multicolumn{6}{|c|}{ Mode number } \\
\hline & & 1 & 2 & 3 & 4 & 5 & 6 \\
\hline $0 \times 0$ & Present & 6.7 & 13.8 & 13.8 & 20.4 & 24.8 & 24.9 \\
\hline \multirow{2}{*}{$L x / 10 \times L y / 10$} & Present & 6.8 & 13.8 & 13.8 & 20.2 & 24.5 & 25.0 \\
\hline & ANSYS & 6.8 & 13.8 & 13.8 & 20.2 & 24.5 & 25.0 \\
\hline$L x / 5 \times L y / 5$ & Present & 6.9 & 13.4 & 13.4 & 19.7 & 24.0 & 26.7 \\
\hline $3 L x / 10 \times 3 L y / 10$ & Present & 7.7 & 12.6 & 12.6 & 19.0 & 23.0 & 29.8 \\
\hline \multirow{2}{*}{$2 L x / 5 \times 2 L y / 5$} & Present & 9.3 & 12.6 & 12.6 & 18.9 & 21.0 & 28.5 \\
\hline & ANSYS & 9.3 & 12.4 & 12.4 & 18.7 & 20.1 & 28.1 \\
\hline$L x / 2 \times L y / 2$ & Present & 12.3 & 14.5 & 14.6 & 20.1 & 20.5 & 27.9 \\
\hline
\end{tabular}

square opening sizes are listed in Table 3 , in which the size of the square cutout varied from $0 \times 0$ to $L x / 2 \times$ $L y / 2$. For the cutout with $0 \times 0$ size, the clamped flexible plate is complete and has no holes. For the other cases in Table 3, the cutout in the clamped plate is square and has free boundary conditions. Simulation results for some cases are also presented to verify the effectiveness of the present method. As shown in Table 3, the cutout size significantly affects the natural frequencies, whose values are increased with the increase of cutout size. The influence trend of the cutout size on the natural frequencies is due to the decrease of the structure quality.

For a specific cutout size, the dynamic characteristic for the flexible plate is mostly determined by the cutout position. The natural frequencies and mode shapes for the clamped plate having one cutout with $1 \mathrm{~m} \times 1 \mathrm{~m}$ size are shown in Table 4 and Figures 4-6, respectively. Here the plates with inner cutout, corner cutout, and edge cutout are presented to show the influence of cutout positions. Both the center of the inner cutout and the original point of the corner cutout are located at $(1 \mathrm{~m}, 1 \mathrm{~m})$. The original and end points of the edge cutout are $(0.5 \mathrm{~m}, 1 \mathrm{~m})$ and $(1 \mathrm{~m}, 1 \mathrm{~m})$, respectively.
The superscripts a and $\mathrm{b}$ in Table 4 indicate the natural frequencies obtained from ANSYS and [18], respectively. Compared with the results from [18], the natural frequencies obtained from the present method compare better with ones obtained from ANSYS. The phenomenon could be observed in Table 4 that the natural frequencies are more sensitive to the cutout positions. As shown in Figures 4-6, the mode shapes are determined by the cutout positions. It can be concluded through the phenomenon in Table 4 and Figures 4-6 that the natural frequency and mode shapes for the first six modes are heavily influenced by the cutout positions.

To widely investigate the influence of the cutouts positions, the first six natural frequencies and correspondingly the mode shapes of the clamped plate having double free cutouts are given in Table 5 and Figures 7-9, respectively. The position distributions of double cutouts with $0.5 \mathrm{~m} \times 0.5 \mathrm{~m}$ size are similar to those considered above. The original points of the double cutouts inside the plate are located at $(0.45 \mathrm{~m}, 0.75 \mathrm{~m})$ and $(1.05 \mathrm{~m}, 0.75 \mathrm{~m})$, respectively. The original points of the double cutouts at the edge of the plate are located at $(0.75 \mathrm{~m}$, $1.5 \mathrm{~m})$ and $(0.75 \mathrm{~m}, 0 \mathrm{~m})$, respectively. The original points of the double cutouts at the corner of the plate are located at 
TABLE 4: Natural frequencies $(\mathrm{Hz})$ for the rectangular plate with a single cutout.

\begin{tabular}{|c|c|c|c|c|c|c|}
\hline Mode number & 1 & 2 & 3 & 4 & 5 & 6 \\
\hline \multirow{3}{*}{ Plate with inner cutout } & 12.4 & 14.5 & 14.6 & 20.0 & 20.2 & 27.7 \\
\hline & $12.4^{\mathrm{a}}$ & $14.4^{\mathrm{a}}$ & $14.4^{\mathrm{a}}$ & $19.5^{\mathrm{a}}$ & $20.2^{\mathrm{a}}$ & $27.5^{\mathrm{a}}$ \\
\hline & $12.4^{\mathrm{b}}$ & $14.9^{\mathrm{b}}$ & $14.9^{\mathrm{b}}$ & $20.5^{\mathrm{b}}$ & $22.6^{\mathrm{b}}$ & $29.4^{\mathrm{b}}$ \\
\hline \multirow{3}{*}{ Plate with edge cutout } & 7.1 & 13.6 & 17.7 & 20.5 & 22.7 & 26.9 \\
\hline & $7.0^{\mathrm{a}}$ & $13.5^{\mathrm{a}}$ & $17.5^{\mathrm{a}}$ & $20.1^{\mathrm{a}}$ & $21.8^{\mathrm{a}}$ & $26.7^{\mathrm{a}}$ \\
\hline & $7.1^{\mathrm{b}}$ & $13.6^{\mathrm{b}}$ & $17.8^{\mathrm{b}}$ & $20.6^{\mathrm{b}}$ & $23.1^{\mathrm{b}}$ & $27.1^{\mathrm{b}}$ \\
\hline \multirow{3}{*}{ Plate with corner cutout } & 6.3 & 12.5 & 15.2 & 22.8 & 23.0 & 26.8 \\
\hline & $6.3^{\mathrm{a}}$ & $12.5^{\mathrm{a}}$ & $15.2^{\mathrm{a}}$ & $22.8^{\mathrm{a}}$ & $23.0^{\mathrm{a}}$ & $26.8^{\mathrm{a}}$ \\
\hline & $6.3^{\mathrm{b}}$ & $12.8^{\mathrm{b}}$ & $15.2^{\mathrm{b}}$ & $23.0^{\mathrm{b}}$ & $23.4^{\mathrm{b}}$ & $26.9^{\mathrm{b}}$ \\
\hline
\end{tabular}

Superscripts $\mathrm{a}$ and $\mathrm{b}$ indicate that the results are obtained from ANSYS and [18], respectively.

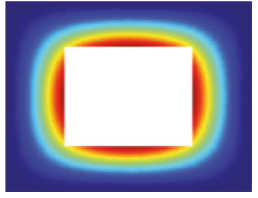

(a)

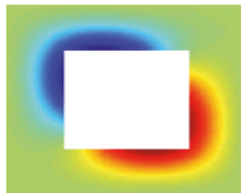

(b)

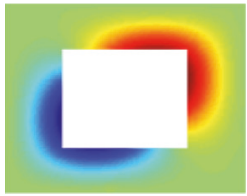

(c)

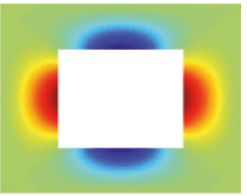

(d)

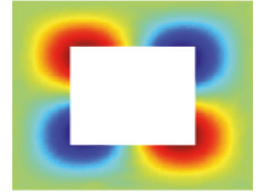

(e)

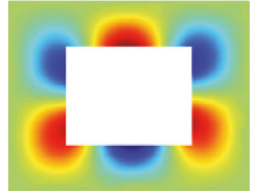

(f)

Figure 4: The first six mode shapes of the square plate with an inner cutout. (a) The first mode; (b) the second mode; (c) the third mode; (d) the fourth mode; (e) the fifth mode; (f) the sixth mode.

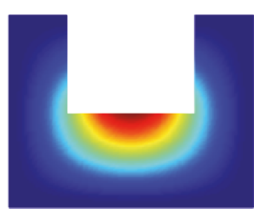

(a)

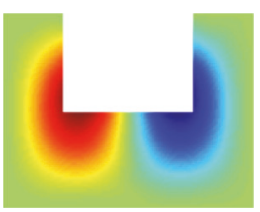

(b)

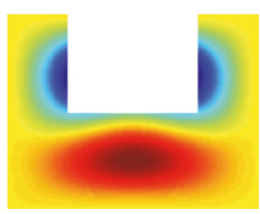

(c)

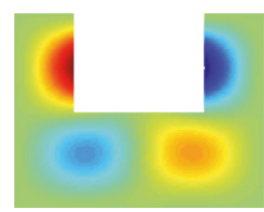

(d)

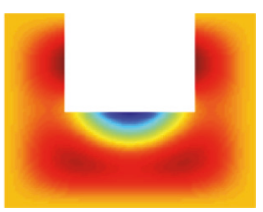

(e)

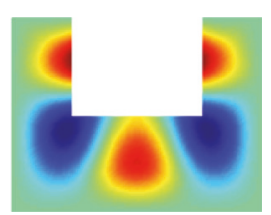

(f)

Figure 5: The first six mode shapes of the square plate with a corner cutout. (a) The first mode; (b) the second mode; (c) the third mode; (d) the fourth mode; (e) the fifth mode; (f) the sixth mode.

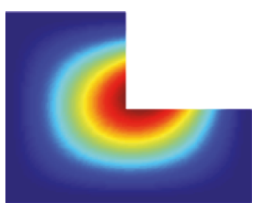

(a)

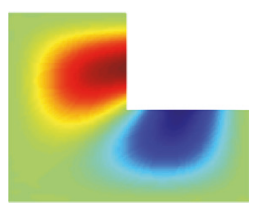

(b)

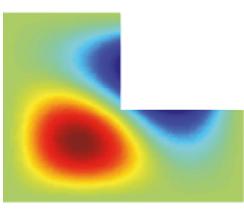

(c)

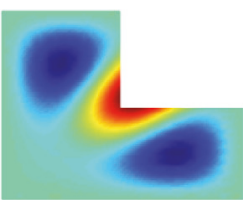

(d)

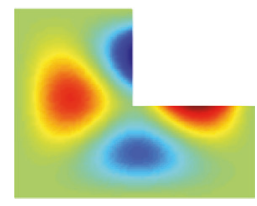

(e)

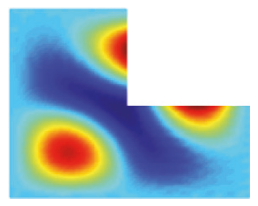

(f)

FIgURE 6: The first six mode shapes of the square plate with an edge cutout. (a) The first mode; (b) the second mode; (c) the third mode; (d) the fourth mode; (e) the fifth mode; (f) the sixth mode.

TABLE 5: Natural frequencies $(\mathrm{Hz})$ for the rectangular plate with double cutouts.

\begin{tabular}{|c|c|c|c|c|c|c|}
\hline Mode number & 1 & 2 & 3 & 4 & 5 & 6 \\
\hline \multirow{2}{*}{ Plate with inner cutouts } & 7.7 & 11.8 & 14.9 & 18.6 & 22.1 & 27.0 \\
\hline & $7.7^{\mathrm{a}}$ & $11.6^{\mathrm{a}}$ & $14.7^{\mathrm{a}}$ & $18.4^{\mathrm{a}}$ & $21.8^{\mathrm{a}}$ & $27.0^{\mathrm{a}}$ \\
\hline \multirow{2}{*}{ Plate with edge cutouts } & 6.2 & 12.4 & 13.2 & 19.2 & 24.1 & 24.5 \\
\hline & $6.2^{\mathrm{a}}$ & $12.2^{\mathrm{a}}$ & $13.2^{\mathrm{a}}$ & $19.1^{\mathrm{a}}$ & $24.0^{\mathrm{a}}$ & $24.4^{\mathrm{a}}$ \\
\hline \multirow{2}{*}{ Plate with corner cutouts } & 6.6 & 13.4 & 13.5 & 19.5 & 24.3 & 24.6 \\
\hline & $6.6^{\mathrm{a}}$ & $13.4^{\mathrm{a}}$ & $13.5^{\mathrm{a}}$ & $19.5^{\mathrm{a}}$ & $24.3^{\mathrm{a}}$ & $24.6^{\mathrm{a}}$ \\
\hline
\end{tabular}

Superscript a indicates the results obtained from ANSYS. 


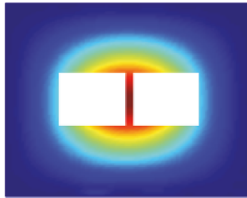

(a)

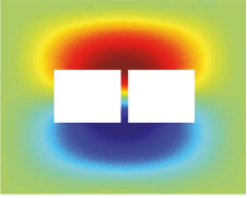

(b)

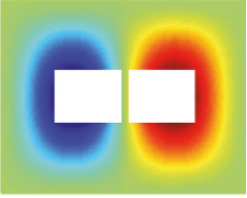

(c)

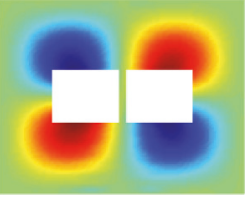

(d)

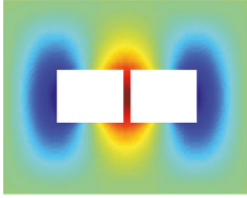

(e)

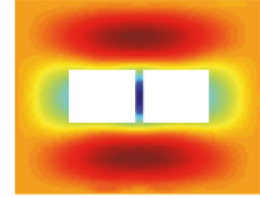

(f)

Figure 7: The first six mode shapes of the square plate with double inner cutouts. (a) The first mode; (b) the second mode; (c) the third mode; (d) the fourth mode; (e) the fifth mode; (f) the sixth mode.

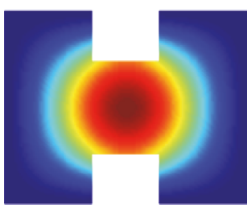

(a)

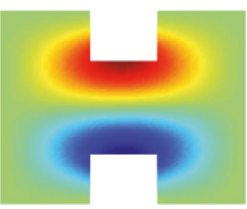

(b)

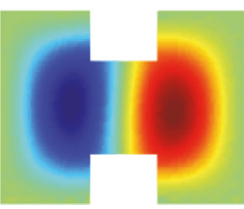

(c)

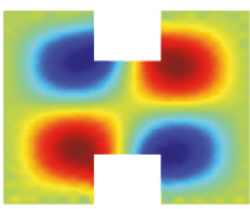

(d)

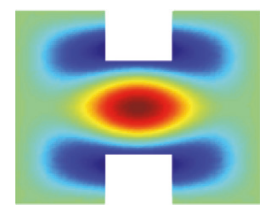

(e)

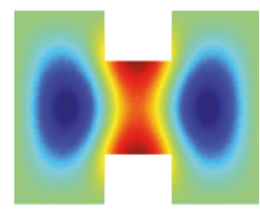

(f)

FiguRE 8: The first six mode shapes of the square plate with double corner cutouts. (a) The first mode; (b) the second mode; (c) the third mode; (d) the fourth mode; (e) the fifth mode; (f) the sixth mode.

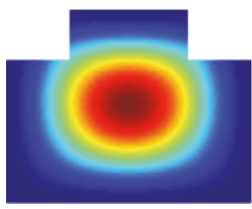

(a)

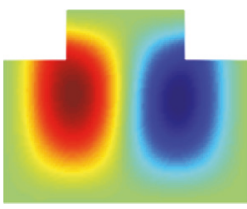

(b)

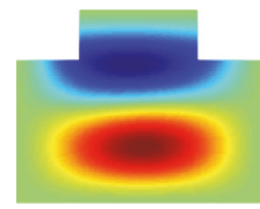

(c)

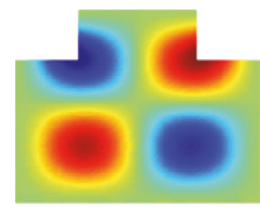

(d)

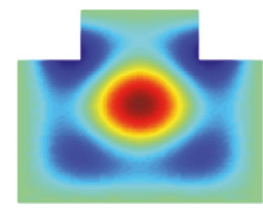

(e)

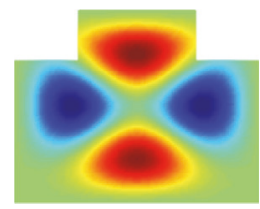

(f)

FIGURE 9: The first six mode shapes of the square plate with double edge cutouts. (a) The first mode; (b) the second mode; (c) the third mode; (d) the fourth mode; (e) the fifth mode; (f) the sixth mode.

TABLE 6: The first six natural frequencies $(\mathrm{Hz})$ for different cutout numbers.

\begin{tabular}{|c|c|c|c|c|c|c|c|}
\hline \multirow{2}{*}{ Cutout number } & \multirow{2}{*}{ Methods } & \multicolumn{6}{|c|}{ Mode number } \\
\hline & & 1 & 2 & 3 & 4 & 5 & 6 \\
\hline \multirow{2}{*}{ One cutout } & Present & 13.1 & 15.7 & 18.7 & 21.8 & 22.2 & 28.4 \\
\hline & ANSYS & 13.1 & 15.6 & 18.7 & 21.7 & 21.9 & 28.1 \\
\hline \multirow{2}{*}{ Two cutouts } & Present & 8.1 & 13.9 & 15.1 & 19.1 & 21.4 & 23.1 \\
\hline & ANSYS & 8.0 & 13.9 & 15.0 & 19.0 & 21.3 & 22.9 \\
\hline \multirow{2}{*}{ Three cutouts } & Present & 7.5 & 9.7 & 14.1 & 15.1 & 19.4 & 20.4 \\
\hline & ANSYS & 7.4 & 9.4 & 14.0 & 14.9 & 19.2 & 20.1 \\
\hline
\end{tabular}

$(0 \mathrm{~m}, 1.5 \mathrm{~m})$ and $(1.5 \mathrm{~m}, 1,5 \mathrm{~m})$, respectively. The superscript a in Table 5 indicates the natural frequencies obtained from ANSYS. As shown in Table 5, the effectiveness of the first six natural frequencies has been validated through the good comparison with the results from ANSYS. It is apparent that the first six natural frequencies and mode shapes for the clamped plate with double cutouts are influenced by the double cutout positions. As the cutout size for this case is smaller, the influence of the double cutout positions is less than that for the case considered above.

The cutout number is an important influence factor for the eignmodes of the flexible plate with cutouts. To clarify the influence rule, the first six natural frequencies and mode shapes for different cutout number are given in Table 6 and Figure 10, respectively. The structural region between $0.4 \mathrm{~m}$ $\leq x \leq 1.5 \mathrm{~m}$ and $0.4 \mathrm{~m} \leq y \leq 1.5 \mathrm{~m}$ is split into one, two, or three cutouts, respectively. For the plate with one free cutout, the size and original point for the inner cutout are $(1.1 \mathrm{~m} \times 1.1 \mathrm{~m})$ and $(0.4 \mathrm{~m}, 0.4 \mathrm{~m})$, respectively. For the plate with double same size free cutouts, the original points of the two cutouts with the length $l x=0.5 \mathrm{~m}$ and the width $l y=1.1 \mathrm{~m}$ are $(0.4 \mathrm{~m}, 0.4 \mathrm{~m})$ and $(1 \mathrm{~m}, 0.4 \mathrm{~m})$, respectively. When the region mentioned above is divided into three same cutouts with the length $l x=0.5 \mathrm{~m}$ and the width $l y=$ $1.1 \mathrm{~m}$, the original points of the three free cutouts are $(0.4 \mathrm{~m}$, $0.4 \mathrm{~m}),(0.8 \mathrm{~m}, 0.4 \mathrm{~m})$, and $(1.2 \mathrm{~m}, 0.4 \mathrm{~m})$, respectively. The most remarkable characteristic in Table 6 is that the natural frequencies decrease with the increase of cutout number. There is an interesting phenomenon in Figure 10 that the second to fifth mode shapes of the plate with one cutout 

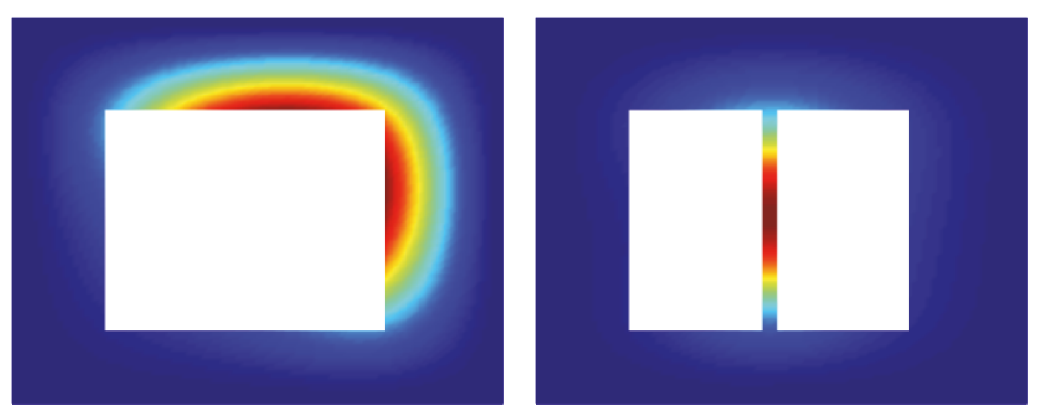

(a)
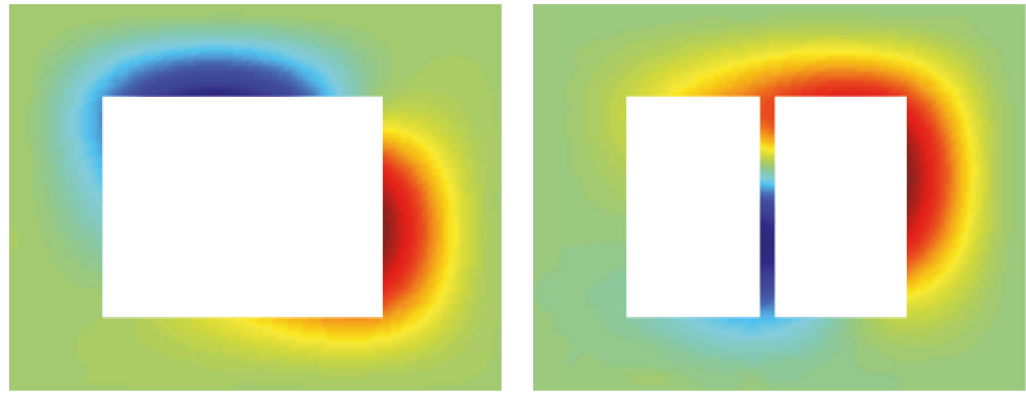

(b)
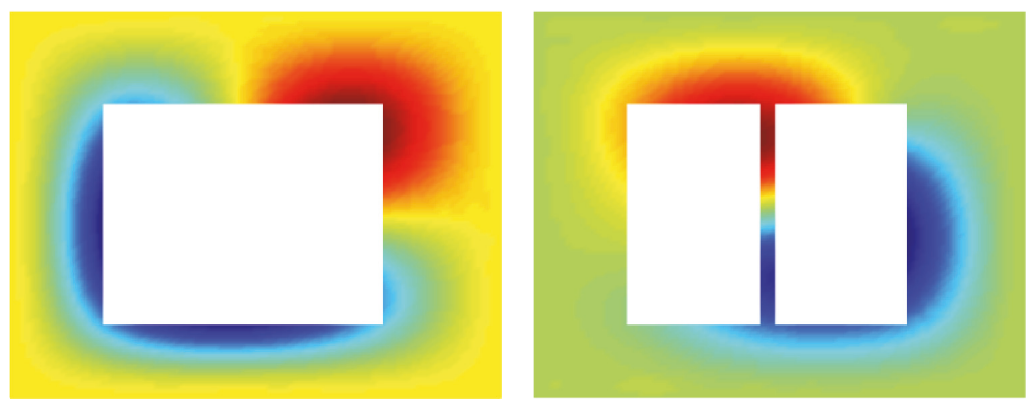

(c)
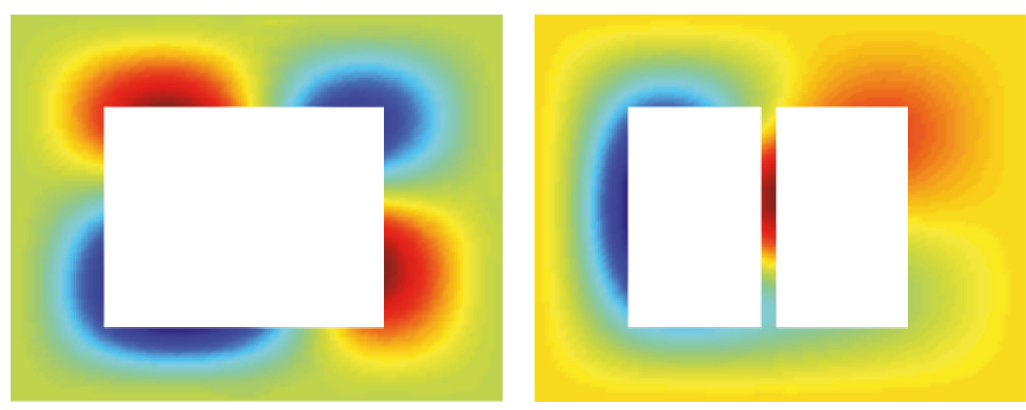

(d)

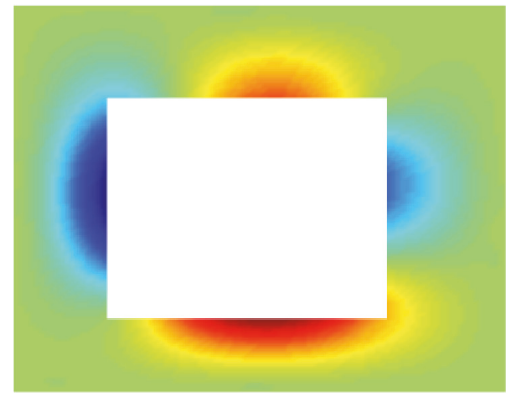

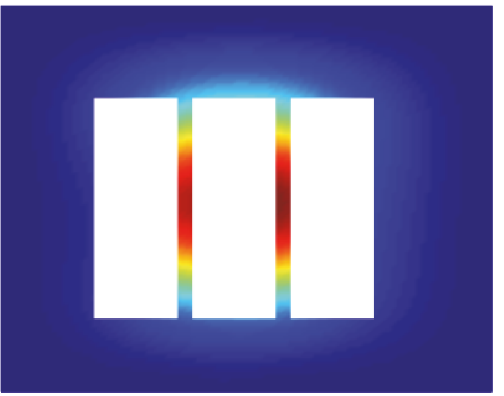
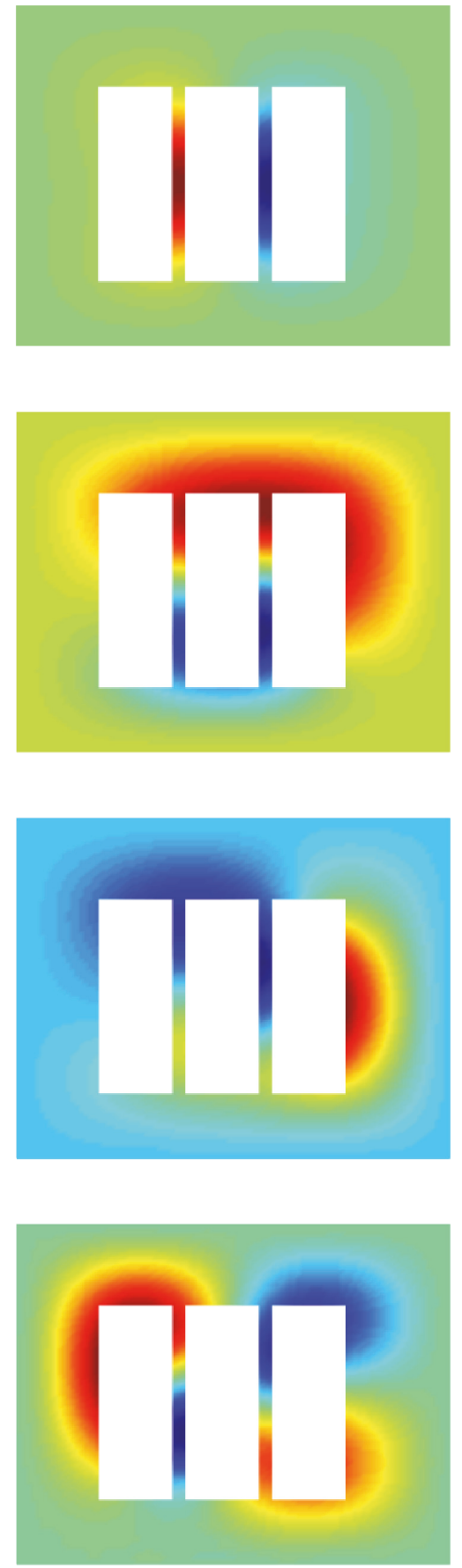

FIgUre 10: Continued. 

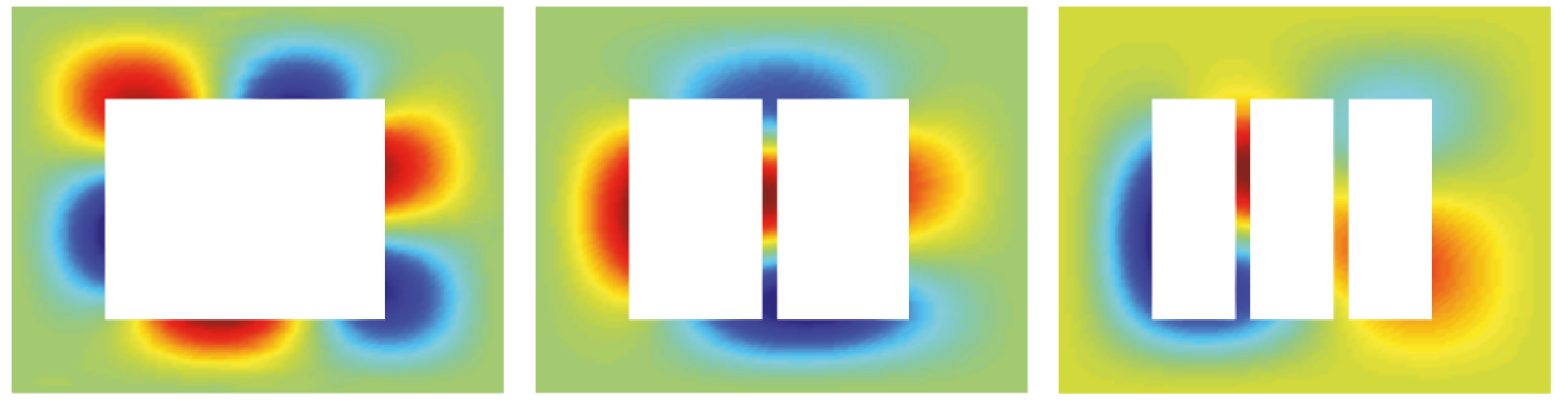

(f)

FIGURE 10: The first six mode shapes for different cutout numbers: the plate with one cutout (the left plot), the plate with two cutouts (the middle plot), and the plate with three cutouts (the right plot).

are in turn similar to the third to sixth mode shapes of the plate with two cutouts. This interesting phenomenon is also appeared between the plate with two cutouts and the plate with three cutouts. Thus, the judgement could be formed from the discussion above that the plate stiffness is influenced by the cutout number.

\section{Conclusion}

This study has clearly demonstrated the possibility of using the energy principles in conjunction with Rayleigh-Ritz solution technique for computing the natural frequencies and modal behavior of plates with rectangular cutouts. The effect of the cutout is taken into account by subtracting the energies of the cutout domains from the total energies of the whole plate with arbitrary boundary conditions. Because the potential energy contributed from the openings boundary has been well-considered, the modeling method proposed in this paper is applicable to the rectangular plate with cutouts having arbitrary boundary conditions including cutouts boundary. Although the rectangular plate is divided into several parts by cutouts, the displacement of the rectangular plate with nonuniform physic parameters is expressed as a unifying expression consisting of a twodimensional trigonometric cosine series and supplementary functions. The energy function of the whole structure is minimized with respect to the unknown Fourier coefficients and the eigenmodes are obtained from the solution of a linear eigenvalue problem. The effectiveness and reliableness of the modeling method are verified through the example of the eigenmodes of the flexible plate with six cutouts. Both the number and position of the openings are variable in the modeling in comparison to other mathematical models. The accuracy of the calculation results of the proposed mathematical model can be improved by the increase of the Fourier truncated series.

The investigation results for different boundary conditions show that the mode frequencies are more influenced by the translational spring stiffness. The influence of the cutout size is mainly reflected on the fundamental frequencies that are increased with the increase of cutout size. The cutout positions have considerable influence on the eigenmodes of the rectangular plate with cutouts, especially for the fundamental frequencies. The study of the cutout number shows that the stiffness of the structure depends on the number of cutouts.

\section{Appendix}

For simplicity, we will introduce the following indices:

$$
\begin{aligned}
& s=m_{p}(M p+1)+n_{p}+1, \\
& t=m_{p}^{\prime}(M p+1)+n_{p}^{\prime}+1 .
\end{aligned}
$$

The submatrices in the first row of $\boldsymbol{K}, \boldsymbol{K}_{i}, \boldsymbol{M}$, and $\boldsymbol{M}_{i}$, which correspond to the derivations of the structural Lagrangian with respect to $A_{m n}^{p}$, can be defined as [19]

$$
\begin{aligned}
& \mathbf{K}_{1-1 ; s, t}=D \int_{0}^{L_{x}} \int_{0}^{L_{y}}\left\{\lambda_{L_{x} m_{p}^{\prime}}^{2} \cos \lambda_{L_{x} m_{p}^{\prime}} x \cos \lambda_{L_{y} n_{p}^{\prime}} y\right. \\
& \cdot \lambda_{L_{x} m_{p}}^{2} \cos \lambda_{L_{x} m_{p}} x \cos \lambda_{L_{y} n_{p}} y+\lambda_{L_{y} n_{p}^{\prime}}^{2} \cos \lambda_{L_{x} m_{p}^{\prime}} x \\
& \cdot \cos \lambda_{L_{y} n_{p}^{\prime}} y \lambda_{L_{y} n_{p}}^{2} \cos \lambda_{L_{x} m_{p}} x \cos \lambda_{L_{y} n_{p}} y \\
& +\mu \lambda_{L_{x} m_{p}^{\prime}}^{2} \cos \lambda_{L_{x} m_{p}^{\prime}} x \cos \lambda_{L_{y} n_{p}^{\prime}} y \lambda_{L_{y} n_{p}}^{2} \cos \lambda_{L_{x} m_{p}} x \\
& \cdot \cos \lambda_{L_{y} n_{p}} y+2(1-\mu) \lambda_{L_{y} n_{p}^{\prime}} \lambda_{L_{x} m_{p}^{\prime}} \sin \lambda_{L_{x} m_{p}^{\prime}} x \\
& \cdot \sin \lambda_{L_{y} n_{p}^{\prime}} y \lambda_{L_{y} n_{p}} \lambda_{L_{x} m_{p}} \sin \lambda_{L_{x} m_{p}} x \\
& \left.\cdot \sin \lambda_{L_{y} n_{p}} y\right\} \mathrm{d} x \mathrm{~d} y+\left[k_{x 0}+(-1)^{m_{p}^{\prime}+m_{p}}\right. \\
& \left.\cdot k_{x L_{x}}\right] \int_{0}^{L_{y}} \cos \lambda_{L_{y} n_{p}^{\prime}} y \cos \lambda_{L_{y} n_{p}} y \mathrm{~d} y+\left[k_{y 0}\right. \\
& \left.+(-1)^{n_{p}^{\prime}+n_{p}} k_{y L_{y}}\right] \int_{0}^{L_{x}} \cos \lambda_{L_{x} m_{p}^{\prime}} x \cos \lambda_{L_{x} m_{p}} x \mathrm{~d} x, \\
& \mathbf{K}_{1-1 ; s, t}^{i}=D \int_{X c i}^{X d i} \int_{Y c i}^{Y d i}\left\{\lambda_{L_{x} m_{p}^{\prime}}^{2} \cos \lambda_{L_{x} m_{p}^{\prime}} x\right. \\
& \cdot \cos \lambda_{L_{y} n_{p}^{\prime}} y \lambda_{L_{x} m_{p}}^{2} \cos \lambda_{L_{x} m_{p}} x \cos \lambda_{L_{y} n_{p}} y+\lambda_{L_{y} n_{p}^{\prime}}^{2} \\
& \cdot \cos \lambda_{L_{x} m_{p}^{\prime}} x \cos \lambda_{L_{y} n_{p}^{\prime}} y \lambda_{L_{y} n_{p}}^{2} \cos \lambda_{L_{x} m_{p}} x
\end{aligned}
$$




$$
\begin{aligned}
& \cdot \cos \lambda_{L_{y} n_{p}} y+\mu \lambda_{L_{x} m_{p}^{\prime}}^{2} \cos \lambda_{L_{x} m_{p}^{\prime}} x \\
& \cdot \cos \lambda_{L_{y} n_{p}^{\prime}} y \lambda_{L_{y} n_{p}}^{2} \cos \lambda_{L_{x} m_{p}} x \cos \lambda_{L_{y} n_{p}} y \\
& +2(1-\mu) \lambda_{L_{y} n_{p}^{\prime}} \lambda_{L_{x} m_{p}^{\prime}} \sin \lambda_{L_{x} m_{p}^{\prime}} x \\
& \cdot \sin \lambda_{L_{y} n_{p}^{\prime}} y \lambda_{L_{y} n_{p}} \lambda_{L_{x} m_{p}} \sin \lambda_{L_{x} m_{p}} x \\
& \left.\cdot \sin \lambda_{L_{y} n_{p}} y\right\} \mathrm{d} x \mathrm{~d} y+\left(k_{x X c i}\right. \\
& \cdot \cos \left(\lambda_{L_{x} m_{p}^{\prime}} X c i\right) \cos \left(\lambda_{L_{x} m_{p}} X c i\right) \\
& +K_{x X c i} \lambda_{L_{x} m_{p}^{\prime}} \lambda_{L_{x} m_{p}} \sin \left(\lambda_{L_{x} m_{p}^{\prime}} X c i\right) \\
& \cdot \sin \left(\lambda_{L_{x} m_{p}} X c i\right)+\cdots+k_{x X d i} \cos \left(\lambda_{L_{x} m_{p}^{\prime}} X d i\right) \\
& \cdot \cos \left(\lambda_{L_{x} m_{p}} X d i\right)+K_{x X d i} \lambda_{L_{x} m_{p}^{\prime}} \lambda_{L_{x} m_{p}} \\
& \left.\cdot \sin \left(\lambda_{L_{x} m_{p}^{\prime}} X d i\right) \sin \left(\lambda_{L_{x} m_{p}} X d i\right)\right) \\
& \times \int_{Y c i}^{Y d i} \cos \lambda_{L_{y} n_{p}^{\prime}} y \cos \lambda_{L_{y} n_{p}} y \mathrm{~d} y+\left(k_{y Y c i}\right. \\
& \cdot \cos \left(\lambda_{L_{y} n_{p}^{\prime}} Y c i\right) \cos \left(\lambda_{L_{y} n_{p}} Y c i\right) \\
& +K_{y Y c i} \lambda_{L_{y} n_{p}^{\prime}} \lambda_{L_{y} n_{p}} \sin \left(\lambda_{L_{y} n_{p}^{\prime}} Y c i\right) \sin \left(\lambda_{L_{y} n_{p}} Y c i\right) \\
& +\cdots+k_{y Y d i} \cos \left(\lambda_{L_{y} n_{p}^{\prime}} Y d i\right) \cos \left(\lambda_{L_{y} n_{p}} Y d i\right) \\
& +K_{y Y d i} \lambda_{L_{y} n_{p}^{\prime}} \lambda_{L_{y} n_{p}} \sin \left(\lambda_{L_{x} m_{p}^{\prime}} Y d i\right) \\
& \left.\cdot \sin \left(\lambda_{L_{x} m_{p}} Y d i\right)\right) \times \int_{X c i}^{X d i} \cos \lambda_{L_{x} m_{p}^{\prime}} x \\
& \cdot \cos \lambda_{L_{x} m_{p}} x \mathrm{~d} x \\
& \mathbf{K}_{1-2 ; s, m_{p}^{\prime}+1}=D \int_{0}^{L_{x}} \int_{0}^{L_{y}}\left\{\lambda_{L_{x} m_{p}^{\prime}}^{2}\right. \\
& \cdot \cos \lambda_{L_{x} m_{p}^{\prime}} x \zeta_{1 L_{y}}(y) \lambda_{L_{x} m_{p}}^{2} \cos \lambda_{L_{x} m_{p}} x \cos \lambda_{L_{y} n_{p}} y \\
& -\cos \lambda_{L_{x} m_{p}^{\prime}} x \zeta_{1 L_{y}}^{\prime \prime}(y) \lambda_{L_{y} n_{p}}^{2} \cos \lambda_{L_{x} m_{p}} x \cos \lambda_{L_{y} n_{p}} y \\
& -\mu \cos \lambda_{L_{x} m_{p}^{\prime}} x \zeta_{1 L_{y}}^{\prime \prime}(y) \lambda_{L_{x} m_{p}}^{2} \cos \lambda_{L_{x} m_{p}} x \\
& \cdot \cos \lambda_{L_{y} n_{p}} y+\mu \lambda_{L_{x} m_{p}^{\prime}}^{2} \cos \lambda_{L_{x} m_{p}^{\prime}} x \zeta_{1 L_{y}}(y) \lambda_{L_{y} n_{p}}^{2} \\
& \cdot \cos \lambda_{L_{x} m_{p}} x \cos \lambda_{L_{y} n_{p}} y-2(1-\mu) \lambda_{L_{x} m_{p}^{\prime}} \zeta_{1 L_{y}}^{\prime}(y) \\
& \cdot \sin \lambda_{L_{x} m_{p}^{\prime}} x \lambda_{L_{y} n_{p}} \lambda_{L_{x} m_{p}} \sin \lambda_{L_{x} m_{p}} x \\
& \left.\cdot \sin \lambda_{L_{y} n_{p}} y\right\} \mathrm{d} x \mathrm{~d} y+\left[k_{x 0}\right. \\
& \left.+(-1)^{m_{p}^{\prime}+m_{p}} k_{x L_{x}}\right] \int_{0}^{L y} \zeta_{1 L_{y}}(y) \cos \lambda_{L_{y} n_{p}} y \mathrm{~d} y
\end{aligned}
$$

$$
\begin{aligned}
& +\left[k_{y 0} \zeta_{1 L_{y}}(0)+(-1)^{n_{p}} k_{y L_{y}} \zeta_{1 L_{y}}\left(L_{y}\right)\right] \\
& \cdot \int_{0}^{L_{x}} \cos \lambda_{L_{x} m_{p}^{\prime}} x \cos \lambda_{L_{x} m_{p}} x \mathrm{~d} x
\end{aligned}
$$$$
\mathbf{K}_{1-2 ; s, m_{p}^{\prime}+1}^{i}=D \int_{X c i}^{X d i} \int_{Y c i}^{Y d i}\left\{\lambda_{L_{x} m_{p}^{\prime}}^{2}\right.
$$$$
\cdot \cos \lambda_{L_{x} m_{p}^{\prime}} x \zeta_{1 L_{y}}(y) \lambda_{L_{x} m_{p}}^{2} \cos \lambda_{L_{x} m_{p}} x \cos \lambda_{L_{y} n_{p}} y
$$$$
-\cos \lambda_{L_{x} m_{p}^{\prime}} x \zeta_{1 L_{y}}^{\prime \prime}(y) \lambda_{L_{y} n_{p}}^{2} \cos \lambda_{L_{x} m_{p}} x \cos \lambda_{L_{y} n_{p}} y
$$$$
-\mu \cos \lambda_{L_{x} m_{p}^{\prime}} x \zeta_{1 L_{y}}^{\prime \prime}(y) \lambda_{L_{x} m_{p}}^{2} \cos \lambda_{L_{x} m_{p}} x
$$$$
\cdot \cos \lambda_{L_{y} n_{p}} y+\mu \lambda_{L_{x} m_{p}^{\prime}}^{2} \cos \lambda_{L_{x} m_{p}^{\prime}} x \zeta_{1 L_{y}}(y) \lambda_{L_{y} n_{p}}^{2}
$$$$
\cdot \cos \lambda_{L_{x} m_{p}} x \cos \lambda_{L_{y} n_{p}} y-2(1-\mu) \lambda_{L_{x} m_{p}^{\prime}} \zeta_{1 L_{y}}^{\prime}(y)
$$$$
\cdot \sin \lambda_{L_{x} m_{p}^{\prime}} x \lambda_{L_{y} n_{p}} \lambda_{L_{x} m_{p}} \sin \lambda_{L_{x} m_{p}} x
$$$$
\left.\cdot \sin \lambda_{L_{y} n_{p}} y\right\} \mathrm{d} x \mathrm{~d} y+\left(k_{x X c i}\right.
$$$$
\cdot \cos \left(\lambda_{L_{x} m_{p}^{\prime}} X c i\right) \cos \left(\lambda_{L_{x} m_{p}} X c i\right)
$$$$
+K_{x X c i} \lambda_{L_{x} m_{p}^{\prime}} \lambda_{L_{x} m_{p}} \sin \left(\lambda_{L_{x} m_{p}^{\prime}} X c i\right)
$$$$
\cdot \sin \left(\lambda_{L_{x} m_{p}} X c i\right)+\cdots+k_{x X d i} \cos \left(\lambda_{L_{x} m_{p}^{\prime}} X d i\right)
$$$$
\cdot \cos \left(\lambda_{L_{x} m_{p}} X d i\right)+K_{x X d i} \lambda_{L_{x} m_{p}^{\prime}} \lambda_{L_{x} m_{p}}
$$$$
\left.\cdot \sin \left(\lambda_{L_{x} m_{p}^{\prime}} X d i\right) \sin \left(\lambda_{L_{x} m_{p}} X d i\right)\right)
$$$$
\times \int_{Y c i}^{Y d i} \zeta_{1 L_{y}}(y) \cos \lambda_{L_{y} n_{p}} y \mathrm{~d} y
$$$$
+\left(k_{y Y c i} \zeta_{1 L_{y}}(Y c i) \cos \left(\lambda_{L_{y} n_{p}} Y c i\right)\right.
$$$$
+K_{y Y c i} \lambda_{L_{y} n_{p}} \zeta_{1 L_{y}}^{\prime}(Y c i) \sin \left(\lambda_{L_{y} n_{p}} Y c i\right)+\cdots
$$$$
+k_{y Y d i} \zeta_{1 L_{y}}(Y d i) \cos \left(\lambda_{L_{y} n_{p}} Y d i\right)
$$$$
\left.+K_{y Y d i} \lambda_{L_{y} n_{p}} \zeta_{1 L_{y}}^{\prime}(Y c i) \sin \left(\lambda_{L_{x} m_{p}} Y d i\right)\right)
$$$$
\times \int_{X c i}^{X d i} \cos \lambda_{L_{x} m_{p}^{\prime}} x \cos \lambda_{L_{x} m_{p}} x \mathrm{~d} x .
$$

We could obtain the submatrices $\mathbf{K}_{1-3 ; s, m_{p}^{\prime}+1}, \mathbf{K}_{1-4 ; s, m_{p}^{\prime}+1}$, $\mathbf{K}_{1-5 ; s, m_{p}^{\prime}+1}$ and $\mathbf{K}_{1-3 ; s, m_{p}^{\prime}+1}^{i}, \mathbf{K}_{1-4 ; s, m_{p}^{\prime}+1}^{i}, \mathbf{K}_{1-5 ; s, m_{p}^{\prime}+1}^{i}$ by replacing the subscript 1 in the $\zeta$-functions in (A.4) and (A.5) with 2, 3, and 4 , respectively.

$$
\begin{gathered}
\mathbf{K}_{1-6 ; s, n_{p}^{\prime}+1}=D \int_{0}^{L_{x}} \int_{0}^{L_{y}}\left\{-\zeta_{1 L_{x}}^{\prime \prime}(x) \cos \lambda_{L_{y} n_{p}^{\prime}} y \lambda_{L_{x} m_{p}}^{2}\right. \\
\cdot \cos \lambda_{L_{x} m_{p}} x \cos \lambda_{L_{y} n_{p}} y+\lambda_{L_{y} n_{p}^{\prime}}^{2} \zeta_{1 L_{x}}(x)
\end{gathered}
$$




$$
\begin{aligned}
& \cdot \cos \lambda_{L_{y} n_{p}^{\prime}} y \lambda_{L_{y} n_{p}}^{2} \cos \lambda_{L_{x} m_{p}} x \cos \lambda_{L_{y} n_{p}} y \\
& +\mu \lambda_{L_{y} n_{p}^{\prime}}^{2} \zeta_{1 L_{x}}(x) \cos \lambda_{L_{y} n_{p}^{\prime}} y \lambda_{L_{x} m_{p}}^{2} \cos \lambda_{L_{x} m_{p}} x \\
& \cdot \cos \lambda_{L_{y} n_{p}} y-\mu \zeta_{1 L_{x}}^{\prime \prime}(x) \cos \lambda_{L_{y} n_{p}^{\prime}} y \lambda_{L_{y} n_{p}}^{2} \\
& \cdot \cos \lambda_{L_{x} m_{p}} x \cos \lambda_{L_{y} n_{p}} y-2(1-\mu) \lambda_{L_{y} n_{p}^{\prime}} \zeta_{1 L_{x}}^{\prime}(x) \\
& \cdot \sin \lambda_{L_{y} n_{p}^{\prime}} y \lambda_{L_{y} n_{p}} \lambda_{L_{x} m_{p}} \sin \lambda_{L_{x} m_{p}} x \\
& \left.\cdot \sin \lambda_{L_{y} n_{p}} y\right\} \mathrm{d} x \mathrm{~d} y+\left[k_{x 0} \zeta_{1 L_{x}}(0)+(-1)^{m_{p}}\right. \\
& \left.\cdot k_{x L_{x}} \zeta_{1 L_{x}}\left(L_{x}\right)\right] \int_{0}^{L_{y}} \cos \lambda_{L_{y} n_{p}^{\prime}} y \cos \lambda_{L_{y} n_{p}} y \mathrm{~d} y \\
& +\left[k_{y 0}+(-1)^{n_{p}^{\prime}+n_{p}} k_{y L_{y}}\right] \int_{0}^{L_{x}} \zeta_{1 L_{x}}(x) \\
& \cdot \cos \lambda_{L_{x} m_{p}} x \mathrm{~d} x \\
& \mathbf{K}_{1-6 ; s, n_{p}^{\prime}+1}^{i}=D \int_{X c i}^{X d i} \int_{Y c i}^{Y d i}\left\{-\zeta_{1 L_{x}}^{\prime \prime}(x) \cos \lambda_{L_{y} n_{p}^{\prime}} y \lambda_{L_{x} m_{p}}^{2}\right. \\
& \cdot \cos \lambda_{L_{x} m_{p}} x \cos \lambda_{L_{y} n_{p}} y+\lambda_{L_{y} n_{p}^{\prime}}^{2} \zeta_{1 L_{x}}(x) \\
& \cdot \cos \lambda_{L_{y} n_{p}^{\prime}} y \lambda_{L_{y} n_{p}}^{2} \cos \lambda_{L_{x} m_{p}} x \cos \lambda_{L_{y} n_{p}} y \\
& +\mu \lambda_{L_{y} n_{p}^{\prime}}^{2} \zeta_{1 L_{x}}(x) \cos \lambda_{L_{y} n_{p}^{\prime}} y \lambda_{L_{x} m_{p}}^{2} \cos \lambda_{L_{x} m_{p}} x \\
& \cdot \cos \lambda_{L_{y} n_{p}} y-\mu \zeta_{1 L_{x}}^{\prime \prime}(x) \cos \lambda_{L_{y} n_{p}^{\prime}} y \lambda_{L_{y} n_{p}}^{2} \\
& \cdot \cos \lambda_{L_{x} m_{p}} x \cos \lambda_{L_{y} n_{p}} y-2(1-\mu) \lambda_{L_{y} n_{p}^{\prime}} \zeta_{1 L_{x}}^{\prime}(x) \\
& \cdot \sin \lambda_{L_{y} n_{p}^{\prime}} y \lambda_{L_{y} n_{p}} \lambda_{L_{x} m_{p}} \sin \lambda_{L_{x} m_{p}} x \\
& \left.\cdot \sin \lambda_{L_{y} n_{p}} y\right\} \mathrm{d} x \mathrm{~d} y+\left(k_{x X c i}\right. \\
& \cdot \cos \left(\lambda_{L_{x} m_{p}^{\prime}} X c i\right) \zeta_{1 L_{x}}(X c i)+K_{x X c i} \lambda_{L_{x} m_{p}} \\
& \cdot \sin \left(\lambda_{L_{x} m_{p}^{\prime}} X c i\right) \zeta_{1 L_{x}}^{\prime}(X c i)+\cdots \\
& +k_{x X d i} \zeta_{1 L_{x}}(X d i) \cos \left(\lambda_{L_{x} m_{p}} X d i\right) \\
& \left.+K_{x X d i} \lambda_{L_{x} m_{p}} \zeta_{1 L_{x}}^{\prime}(X d i) \sin \left(\lambda_{L_{x} m_{p}} X d i\right)\right) \\
& \times \int_{Y c i}^{Y d i} \cos \lambda_{L_{y} n_{p}^{\prime}} y \cos \lambda_{L_{y} n_{p}} y \mathrm{~d} y+\left(k_{y Y c i}\right. \\
& \cdot \cos \left(\lambda_{L_{y} n_{p}^{\prime}} Y c i\right) \cos \left(\lambda_{L_{y} n_{p}} Y c i\right) \\
& +K_{y Y c i} \lambda_{L_{y} n_{p}^{\prime}} \lambda_{L_{y} n_{p}} \sin \left(\lambda_{L_{y} n_{p}^{\prime}} Y c i\right) \sin \left(\lambda_{L_{y} n_{p}} Y c i\right) \\
& +\cdots+k_{y Y d i} \cos \left(\lambda_{L_{y} n_{p}^{\prime}} Y d i\right) \cos \left(\lambda_{L_{y} n_{p}} Y d i\right)
\end{aligned}
$$

$$
\begin{aligned}
& +K_{y Y d i} \lambda_{L_{y} n_{p}^{\prime}} \lambda_{L_{y} n_{p}} \sin \left(\lambda_{L_{x} m_{p}^{\prime}} Y d i\right) \\
& \left.\cdot \sin \left(\lambda_{L_{x} m_{p}} Y d i\right)\right) \times \int_{X c i}^{X d i} \zeta_{1 L_{x}}(x) \cos \lambda_{L_{x} m_{p}} x \mathrm{~d} x .
\end{aligned}
$$

We could obtain the submatrices $\mathbf{K}_{1-7 ; s, n_{p}^{\prime}+1}, \mathbf{K}_{1-8 ; s, n_{p}^{\prime}+1}$, $\mathbf{K}_{1-9 ; s, n_{p}^{\prime}+1}$ and $\mathbf{K}_{1-7 ; s, n_{p}^{\prime}+1}^{i}, \mathbf{K}_{1-8 ; s, n_{p}^{\prime}+1}^{i}, \mathbf{K}_{1-9 ; s, n_{p}^{\prime}+1}^{i}$ by replacing the subscript 1 in the $\zeta$-functions in (A.6) and (A.7) with 2, 3, and 4 , respectively.

$$
\begin{gathered}
\mathbf{M}_{1-1 ; s, t}=\rho h \int_{0}^{L_{x}} \int_{0}^{L_{y}} \cos \lambda_{L_{x} m_{p}^{\prime}} x \cos \lambda_{L_{y} n_{p}^{\prime}} y \\
\cdot \cos \lambda_{L_{x} m_{p}} x \cos \lambda_{L_{y} n_{p}} y \mathrm{~d} x \mathrm{~d} y, \\
\mathbf{M}_{1-1 ; s, t}^{i}=\rho h \int_{X c i}^{X d i} \int_{Y c i}^{Y d i} \cos \lambda_{L_{x} m_{p}^{\prime}} x \cos \lambda_{L_{y} n_{p}^{\prime}} y \\
\cdot \cos \lambda_{L_{x} m_{p}} x \cos \lambda_{L_{y} n_{p}} y \mathrm{~d} x \mathrm{~d} y, \\
\mathbf{M}_{1-2 ; s, m_{p}^{\prime}+1}=\rho h \int_{0}^{L_{x}} \int_{0}^{L_{y}} \cos \lambda_{L_{x} m_{p}^{\prime}} x \zeta_{1 L_{y}}(y) \\
\cdot \cos \lambda_{L_{x} m_{p}} x \cos \lambda_{L_{y} n_{p}} y \mathrm{~d} x \mathrm{~d} y, \\
\mathbf{M}_{1-2 ; s, m_{p}^{\prime}+1}^{i}=\rho h \int_{X c i}^{X d i} \int_{Y c i}^{Y d i} \cos \lambda_{L_{x} m_{p}^{\prime}} x \zeta_{1 L_{y}}(y) \\
\cdot \cos \lambda_{L_{x} m_{p}} x \cos \lambda_{L_{y} n_{p}} y \mathrm{~d} x \mathrm{~d} y .
\end{gathered}
$$

The submatrices $\mathbf{M}_{1-3 ; s, m_{p}^{\prime}+1}, \mathbf{M}_{1-4 ; s, m_{p}^{\prime}+1}, \mathbf{M}_{1-5 ; s, m_{p}^{\prime}+1}$ and $\mathbf{M}_{1-3 ; s, m_{p}^{\prime}+1}^{i}, \mathbf{M}_{1-4 ; s, m_{p}^{\prime}+1}^{i}, \mathbf{M}_{1-5 ; s, m_{p}^{\prime}+1}^{i}$ could be obtained by replacing the subscript 1 in the $\zeta$-functions in (A.10) and (A.11) with 2,3 , and 4 , respectively.

$$
\begin{aligned}
& \mathbf{M}_{1-6 ; s, n_{p}^{\prime}+1}=\rho h \int_{0}^{L_{x}} \int_{0}^{L_{y}} \cos \lambda_{L_{y} n_{p}^{\prime}} y \zeta_{1 L_{x}}(x) \\
& \cdot \cos \lambda_{L_{x} m_{p}} x \cos \lambda_{L_{y} n_{p}} y \mathrm{~d} x \mathrm{~d} y, \\
& \mathbf{M}_{1-6 ; s, n_{p}^{\prime}+1}^{i}=\rho h \int_{X c i}^{X d i} \int_{Y c i}^{Y d i} \cos \lambda_{L_{y} n_{p}^{\prime}} y \zeta_{1 L_{x}}(x) \\
& \cdot \cos \lambda_{L_{x} m_{p}} x \cos \lambda_{L_{y} n_{p}} y \mathrm{~d} x \mathrm{~d} y .
\end{aligned}
$$

The submatrices $\mathbf{M}_{1-7 ; s, n_{p}^{\prime}+1}, \mathbf{M}_{1-8 ; s, n_{p}^{\prime}+1}, \mathbf{M}_{1-9 ; s, n_{p}^{\prime}+1}$ and $\mathbf{M}_{1-7 ; s, n_{p}^{\prime}+1}^{i}, \mathbf{M}_{1-8 ; s, n_{p}^{\prime}+1}^{i}, \mathbf{M}_{1-9 ; s, n_{p}^{\prime}+1}^{i}$ could be obtained by replacing the subscript 1 in the $\zeta$-functions in (A.12) and (A.13) with 2,3 , and 4 , respectively.

\section{Conflicts of Interest}

The authors declare that they have no conflicts of interest.

\section{Acknowledgments}

The authors gratefully acknowledge the financial support from the National Natural Science Foundation of China 
(nos. 51705071, 51475088, and 51775125), Natural Science Foundation of Jilin Province of China (no. 20140101065JC), the Fundamental Research Funds for the Central Universities of China (no. HEUCFG201713), and the Research Foundation of Education Department of Jilin Province, China (no. 2015244).

\section{References}

[1] D. R. Avalos, H. A. Larrondo, P. A. A. Laura, and R. E. Rossi, "Transverse vibrations of simply supported rectangular plates with rectangular cutouts carrying an elastically mounted concentrated mass," Journal of Sound and Vibration, vol. 202, no. 4, pp. 585-592, 1997.

[2] D. R. Avalos and P. A. A. Laura, "Transverse vibrations of simply supported rectangular plates with two rectangular cutouts," Journal of Sound and Vibration, vol. 267, no. 4, pp. 967-977, 2003.

[3] S. Takahashi, "Vibration of Rectangular Plates with Circular Holes," Journal of the Japan Society of Mechanical Engineers, vol. 1, no. 4, pp. 380-385, 1958.

[4] P. Paramasivam, "Free vibration of square plates with square openings," Journal of Sound and Vibration, vol. 30, no. 2, pp.173$178,1973$.

[5] R. Ali and S. J. Atwal, "Prediction of natural frequencies of vibration of rectangular plates with rectangular cutouts," Computers \& Structures, vol. 12, no. 6, pp. 819-823, 1980.

[6] J. N. Reddy, "Large amplitude flexural vibration of layered composite plates with cutouts," Journal of Sound and Vibration, vol. 83, no. 1, pp. 1-10, 1982.

[7] K. Y. Lam, K. C. Hung, and S. T. Chow, "Vibration analysis of plates with cutouts by the modified Rayleigh-Ritz method," Applied Acoustics, vol. 28, no. 1, pp. 49-60, 1989.

[8] D. T. Huang, "Effects of constraint, circular cutout and in-plane loading on vibration of rectangular plates," International Journal of Mechanical Sciences, vol. 68, pp. 114-124, 2013.

[9] A. K. L. Srivastava, P. K. Datta, and A. M. Sheikh, "Transverse vibration of stiffened plates with cutouts subjected to inplane uniform edge loading at the plate boundary," Shock and Vibration, vol. 11, no. 1, pp. 9-19, 2004.

[10] S. S. Hota and P. Padhi, "Vibration of plates with arbitrary shapes of cutouts," Journal of Sound and Vibration, vol. 302, no. 4-5, pp. 1030-1036, 2007.

[11] G. Aksu and R. Ali, "Determination of dynamic characteristics of rectangular plates with cutouts using a finite difference formulation," Journal of Sound and Vibration, vol. 44, no. 1, pp. 147-158, 1976.

[12] M. K. Kwak and S. Han, "Free vibration analysis of rectangular plate with a hole by means of independent coordinate coupling method," Journal of Sound and Vibration, vol. 306, no. 1-2, pp. 12-30, 2007.

[13] G. Mundkur, R. B. Bhat, and S. Neriya, "Vibration of plates with cut-outs using boundary characteristic orthogonal polynomial functions in the Rayleigh-Ritz method," Journal of Sound and Vibration, vol. 176, no. 1, pp. 136-144, 1994.

[14] D. Wang, "Sensitivity analysis and shape optimization of a hole in a vibrating rectangular plate for eigenfrequency maximization," Journal of Engineering Mechanics, vol. 138, no. 6, pp. 662674, 2012.

[15] P. A. A. Laura, E. Romanelli, and R. E. Rossi, "Transverse vibrations of simply supported rectangular plates with rectangular cutouts," Journal of Sound and Vibration, vol. 202, no. 2, pp. 275283, 1997.

[16] R. H. Gutierrez, P. A. A. Laura, and C. A. Rossit, "Fundamental frequency of transverse vibration of a clamped rectangular orthotropic plate with a free-edge hole," Journal of Sound and Vibration, vol. 235, no. 4, pp. 697-701, 2000.

[17] M. Huang and T. Sakiyama, "Free vibration analysis of rectangular plates with variously-shaped holes," Journal of Sound and Vibration, vol. 226, no. 4, pp. 769-786, 1999.

[18] Y. Chen, G. Jin, and Z. Liu, "Flexural and in-plane vibration analysis of elastically restrained thin rectangular plate with cutout using Chebyshev-Lagrangian method," International Journal of Mechanical Sciences, vol. 89, pp. 264-278, 2014.

[19] S. X. Shi, G. Y. Jin, and Z. G. Liu, "Vibro-acoustic behaviors of an elastically restrained double-panel structure with an acoustic cavity of arbitrary boundary impedance," Applied Acoustics, vol. 76, pp. 431-444, 2014. 


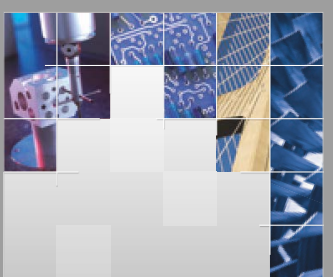

\section{Enfincering}
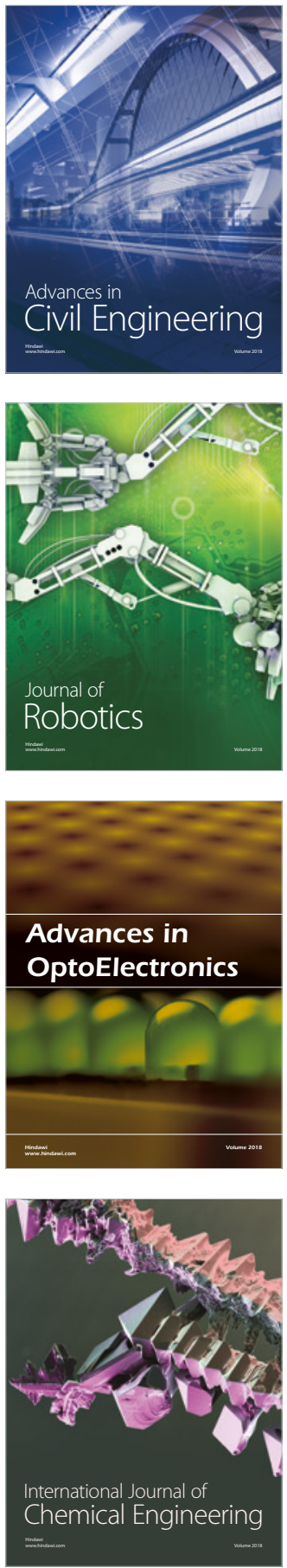

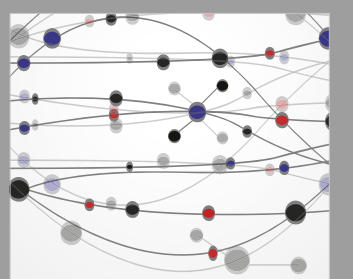

\section{Rotating \\ Machinery}

The Scientific World Journal

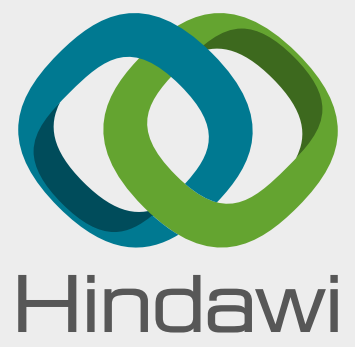

Submit your manuscripts at

www.hindawi.com
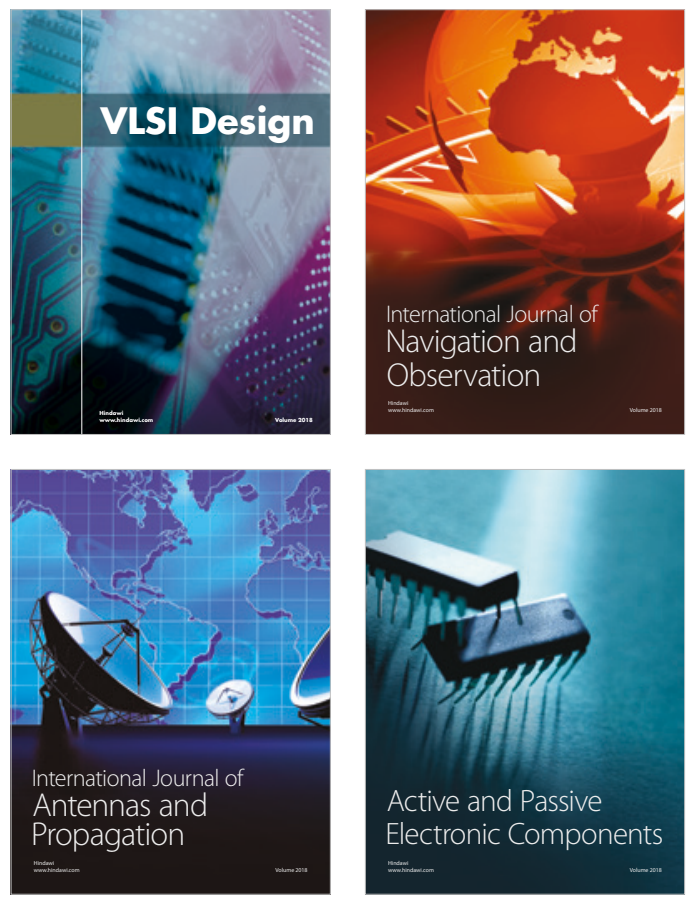
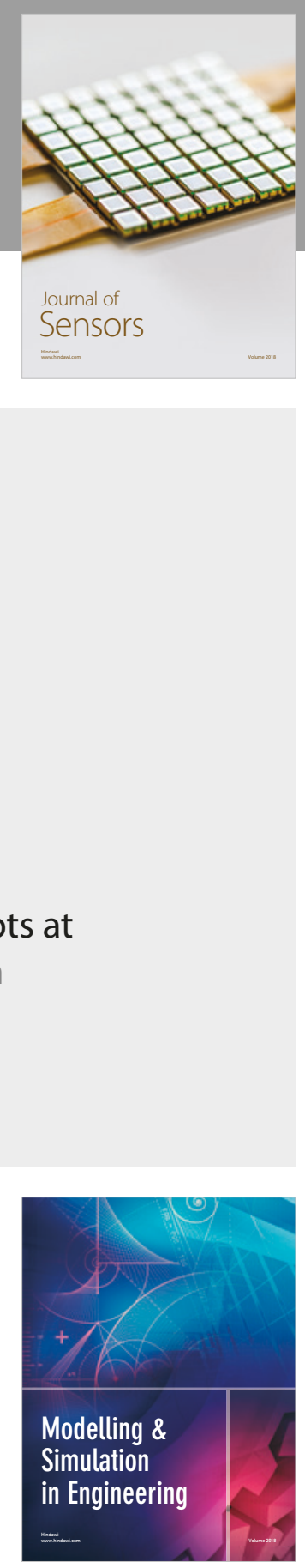

\section{Advances \\ Multimedia}
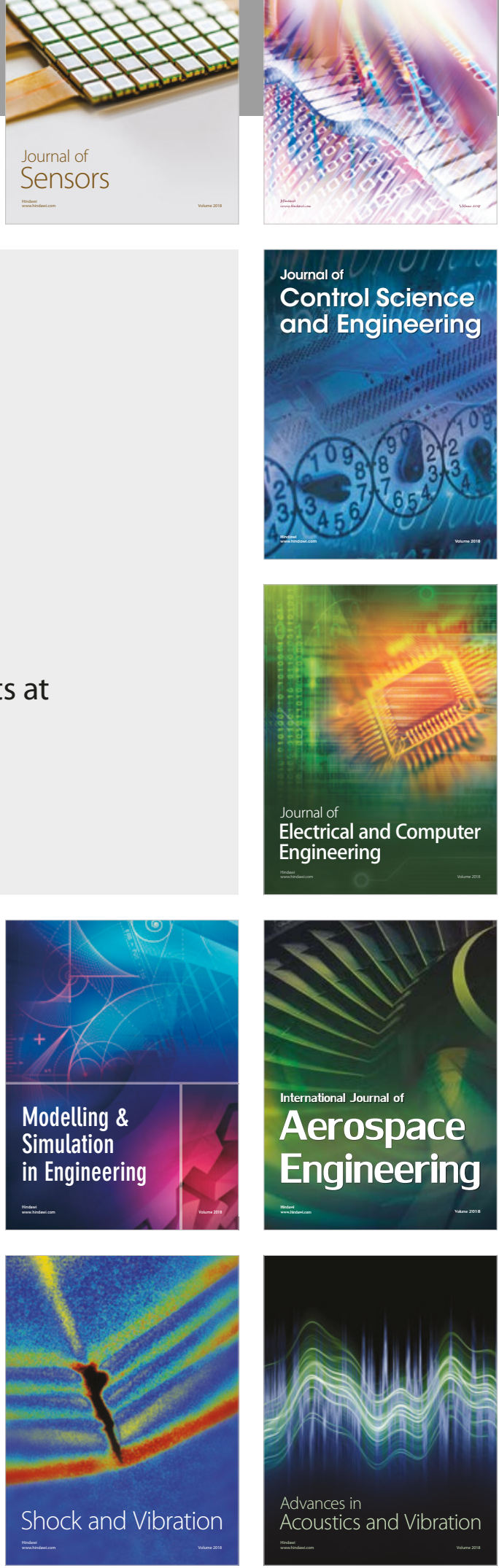